OPEN ACCESS

Edited by:

Muhammad E. H. Chowdhury,

Qatar University, Qatar

Reviewed by:

Abid Ali Khan,

Aligarh Muslim University, India

Yiyun Lan,

Northwestern University,

United States

*Correspondence:

Josefina Gutierrez-Martinez

josefina_gutierrez@hotmail.com

tThese authors have contributed equally to this work and share first

authorship

Specialty section:

This article was submitted to

Neuroprosthetics,

a section of the journal

Frontiers in Neuroscience

Received: 29 April 2021 Accepted: 09 December 2021

Published: 21 January 2022

Citation:

Mercado-Gutierrez JA

Dominguez R, Hernandez-Popo I,

Quinzaños-Fresnedo J,

Vera-Hernandez A, Leija-Salas $L$ and Gutierrez-Martinez J (2022) A Flexible

Pulse Generator Based on a Field

Programmable Gate Array

Architecture for Functional Electrical

Stimulation

Front. Neurosci. 15:702781. doi: 10.3389/fnins.2021.702781

\section{A Flexible Pulse Generator Based on a Field Programmable Gate Array Architecture for Functional Electrical Stimulation}

\author{
Jorge A. Mercado-Gutierrez ${ }^{1,2+}$, Ricardo Dominguez³, Ignacio Hernandez-Popo 4 , \\ Jimena Quinzaños-Fresnedo ${ }^{5}$, Arturo Vera-Hernandez ${ }^{1}$, Lorenzo Leija-Salas ${ }^{1}$ and \\ Josefina Gutierrez-Martinez ${ }^{2 *}$
}

\begin{abstract}
' Departamento de Ingeniería Eléctrica, Sección Bioelectrónica, Centro de Investigación y de Estudios Avanzados del Instituto Politécnico Nacional, Mexico City, Mexico, ${ }^{2}$ División de Investigación en Ingeniería Médica, Instituto Nacional de Rehabilitación Luis Guillermo Ibarra Ibarra, Mexico City, Mexico, ${ }^{3}$ Departamento de Ingeniería Eléctrica, Universidad Autónoma Metropolitana - Iztapalapa, Mexico City, Mexico, ${ }^{4}$ CONACYT - Instituto Nacional de Rehabilitación Luis Guillermo Ibarra Ibarra, Mexico City, Mexico, ${ }^{5}$ División de Rehabilitación Neurológica, Instituto Nacional de Rehabilitación Luis Guillermo Ibarra Ibarra, Mexico City, Mexico
\end{abstract}

Non-invasive Functional Electrical Stimulation (FES) is a technique applied for motor rehabilitation of patients with central nervous system injury. This technique requires programmable multichannel systems to configure the stimulation parameters (amplitude, frequency, and pulse width). Most FES systems are based on microcontrollers with fixed architecture; this limits the control of the parameters and the scaling to multiple channels. Although field programmable gate arrays (FPGA) have been used in FES systems as alternative to microcontrollers, most of them focus on signal acquisition, processing, or communication functions, or are for invasive stimulation. A few FES systems report using FPGAs for parameter configuration and pulse generation in non-invasive FES. However, generally they limit the value of the frequency or amplitude parameters to enable multichannel operation. This restricts free selection of parameters and implementation of modulation patterns, previously reported to delay FES-induced muscle fatigue. To overcome those limitations, this paper presents a proof-of-concept (technology readiness level three-TRL 3) regarding the technical feasibility and potential use of an FPGA-based pulse generator for noninvasive FES applications (PG-nFES). The main aims were: (1) the development of a flexible pulse generator for FES applications and (2) to perform a proof-of-concept of the system, comprising: electrical characterization of the stimulation parameters, and verification of its potential for upper limb FES applications. Biphasic stimulation pulses with high linearity $\left(r^{2}>0.9998\right)$ and repeatability $(>0.81)$ were achieved by combining the PG-nFES with a current-controlled output stage. Average percentage error in the characterizations was under 3\% for amplitude (1-48 mA) and pulse width (20-400 $\mu \mathrm{s})$, and $0 \%$ for frequency $(10-150 \mathrm{~Hz})$. A six-channel version of the PG-nFES was implemented to demonstrate the scalability feature. The independence of parameters was tested with three patterns of co-modulation of two parameters. Moreover, two 
complete FES channels were implemented and the claimed features of the PG-nFES were verified by performing upper limb functional movements involving the hand and the arm. Finally, the system enabled implementation of a stimulation pattern with comodulation of frequency and pulse width, applied successfully for efficient elbow during repetitions of a functional movement.

Keywords: field programmable gate array, functional electric stimulation, bioelectronics, frequency modulation, neurorehabilitation, upper limb, neuroprosthesis, muscle fatigue

\section{INTRODUCTION}

In recent years, several rehabilitation strategies have been used to address the motor sequelae of brain and spinal cord injuries. One example is the Functional Electrical Stimulation (FES) technique, used in neurological rehabilitation programs to improve motor function and manual dexterity (Popovic et al., 2001). This technique is based on the application of low intensity, biphasic electrical impulses that produce action potentials in peripheral nerves and generate muscle contractions (Eraifej et al., 2017). The literature reports the use of FES systems as an effective approach to induce functional rehabilitation of the upper limb (Quandt and Hummel, 2014; Osuagwu et al., 2016). The design of electrical stimulation systems has evolved to fulfill the growing needs of FES applications, from dedicated customed-designed analog control circuitry (Vodovnik et al., 1967), to configurable, highspeed multifunction digital systems (Kutlu et al., 2017) which employ highly integrated programmable devices.

Most FES systems reported in the research literature (Chung et al., 2004; Popovic et al., 2005; Jovičić et al., 2012; Luzio de Melo et al., 2015; Venugopalan et al., 2015) are based on microcontrollers. These devices manage the configuration and timing of the stimulation parameters: amplitude, frequency and pulse-width (Stewart et al., 2016). However, microcontrollers have a fixed and low number of timers and of pulse-width modulation blocks, and they execute one instruction at a time. Those features limit the design of FES systems (Stewart et al., 2016) regarding the number of stimulation channels and their flexibility for independent control of stimulation parameters, two important features in FES applications (Kesar et al., 2008; Grimm and Gharabaghi, 2016). In the other hand, there are design strategies for FES systems that use programmable devices known as field programmable gate arrays (FPGAs). The main advantage of using an FPGA for this end is the large number of logic blocks arrays and interconnections, which are programmable and reconfigurable. FPGAs allow the implementation and almost simultaneous execution of several complex digital functions. Additionally, they require a small board space, are energy efficient and have a low non-recurring engineering cost. Therefore, FPGAs have enabled the design of customized digital electronic systems for several applications, such as digital control, communication interfaces, signals and image processing, computer algorithms, machine learning, and big data (Ruiz-Rosero et al., 2019).

From the 1990s, FPGAs have been used to develop FES systems. They have been applied for Peripheral Nervous System (PNS) stimulation, like bladder (Sawan et al., 1996; Arabi and
Sawan, 1999), respiration (Zbrzeski et al., 2016; Castelli et al., 2017), and visual prostheses (Wong et al., 2005). Also, they have been used for Central Nervous System (CNS) stimulation, including intracortical (Sugiura et al., 2016) and deep brain stimulation (Karami et al., 2016). Moreover, FPGAs have been applied to implement various functions in FES systems, such as data communication (Thanasoulis et al., 2012), signal acquisition (Hsueh et al., 2015), signal processing (Ying et al., 2007; Hsueh et al., 2015; Ranganathan et al., 2019), and control (Ambroise et al., 2013; Karami et al., 2016; Shahdoost et al., 2017).

A key function in FES systems is the generation of stimulation pulses and the control of their main parameters: pulse duration, amplitude, and frequency. These parameters largely determine the activation of the target biological structures, and they need to be adapted for each stimulation channel, according to the context of the FES application. Thus, FPGAs can be useful to implement the required functions for configuration and management of stimulation parameters in an FES system. FES systems using FPGAs for the generation and control of stimulation pulses have been reported since the 1990s (Sawan et al., 1996) up to very recently (Ranganathan et al., 2019). However, most of them were designed for invasive applications, with pulse amplitude values in the range of hundreds of $\mu \mathrm{A}$ (Amerijckx et al., 1998; Castelli et al., 2017) to a few mA (Sawan et al., 1996; Simpson and Ghovanloo, 2007). In contrast, non-invasive FES applications require larger electrodes (up to tens of $\mathrm{cm}^{2}$ ) placed over the skin (transcutaneous), which increase the pulse amplitude requirements: from a few to tens of $\mathrm{mA}$.

There are few reports of transcutaneous (non-invasive) electrical stimulation systems utilizing FPGAs (Benzaba et al., 2008; Chen et al., 2009; Annetta et al., 2019). Benzaba et al. (2008) presented the design of a Transcutaneous Electrical Nerve Stimulation (TENS) system, using an FPGA for the control of parameters of monophasic stimulation pulses. However, they presented only computer simulations and preliminary tests of the system, which did not demonstrate the actual functionality of the system. A year later, Chen et al. (2009) developed a FES system for eyelid reanimation in patients with facial paralysis. They used an FPGA for signal processing of EMG signals to detect eye blinks and send activation commands to a programmable 8-channel digitally controlled current stimulator, implemented on an Application Specific Integrated Circuit (ASIC). That system generated biphasic stimulation pulses with maximum amplitude of $6 \mathrm{~mA}$.

More recently, Annetta et al. (2019) reported a multichannel FES system based on two FPGA circuits. This system controls 
an array of small stimulation electrodes over the forearm, which produce a variety of movements of the fingers, wrist and forearm. However, they used a monophasic pulse shape to simplify the design, acknowledging the convenience of biphasic stimulation pulses for safer, long-term interventions. Moreover, their wired communication interface constrained its portability and limited the stimulation pulse rate to a maximum of 50 pulses per second (divided in the number of active channels at a time). Also, the maximum stimulation amplitude was $20 \mu \mathrm{A}$, which would prevent their use with standard surface stimulation electrodes $\left(10-50 \mathrm{~cm}^{2}\right)$, used in FES clinical practice.

Previous FPGA-based FES systems have not taken full advantage of their programmable core device, to allow high flexibility in the configuration of stimulation parameters. On the contrary, they often limit some parameters to fixed (amplitude or frequency) or dependent values (frequency) among different channels. Furthermore, this restrains the development and use of electrical stimulation patterns comprising modulation of parameters, a relevant approach for minimizing muscle fatigue during the use of FES (Ibitoye et al., 2016). Early muscle fatigue is a great limiting factor for the use of FES interventions aimed to motor rehabilitation of people with neurological conditions (Thrasher et al., 2005), reducing the stimulation time and the potential therapeutic effects. Several approaches have been proposed in the past for modulation of one or more parameters, in order to decrease reduce or delay fatigue and to enhance the performance of FES applications (Ibitoye et al., 2016).

Commercial electrical stimulation systems often allow modulation of a single stimulation parameter at a time: pulse width or amplitude. However, previous works have suggested that co-modulation of two (Gregory et al., 2007; Chou et al., 2008; Downey et al., 2011) or more (Nekoukar, 2021) stimulation parameters can help to reduce early FES-induced muscle fatigue. However, some of those works required the implementation of custom hardware solutions to obtain the desired stimulation patterns (Downey et al., 2011; Nekoukar, 2021). Furthermore, these solutions are aimed to single-channel stimulation of the quadriceps muscle (Gregory et al., 2007; Kesar et al., 2008; Downey et al., 2011). Thus, they are not optimal for clinical research in FES applications, for example, of the upper limb.

Taking into consideration the previous ideas, there is a niche for the design of configurable electrical stimulation systems using an FPGA to handle the pulse generation function, with flexible control of stimulation parameters suitable for non-invasive FES applications, and a scalable architecture to multiple stimulation channels. The main aims of this work are: (1) to develop the prototype of a pulse generator for non-invasive FES based on an FPGA (PG-nFES) and (2) to perform a proof-of-concept of the system, comprising the electrical test bench characterization of the stimulation parameters of the full range stimulation parameters and the verification of the system's potential for transcutaneous FES applications.

The design of the PG-nFES takes advantage of FPGA features to integrate, in a single device, a flexible digital pulse generator for biphasic stimulation, the device control logic, and a serial communication interface for configuration of the stimulation parameters. Moreover, the architecture of the PGnFES is designed to be scalable, a highly desirable feature for the design of multichannel electrical stimulation systems for FES applications.

\section{MATERIALS AND METHODS}

\section{Development of the Pulse Generator for Non-invasive FES Applications}

The development of the pulse generator for non-invasive FES (PG-nFES) is divided in three stages: first, the identification of critical design specifications, second, the design and implementation of the system, and third, the characterization and proof-of-concept of the system of the system. These stages are described below.

\section{Design Specifications}

The main technical specifications for the design of the PG-nFES are summarized in Table $\mathbf{1 .}$

\section{Field Programmable Gate Arrays Features and Programming}

To implement the PG-nFES, a Spartan ${ }^{\circledR}-6$ XC6SLX9-2TQG144I FPGA (Xilinx ${ }^{\circledR}$ Inc., San Jose CA, United States) was used, containing 715 Configurable Logic Blocks, 9152 gates and 576 $\mathrm{kb}$ of RAM. This device is embedded in the Mojo v3, an FPGA prototyping board with a main clock frequency of $50 \mathrm{MHz}, 84$ digital input/output pins, and an ATMEGA32U4 microcontroller for programming the FPGA, analog signal acquisition and digital interfacing.

The FPGA code was written in the ISE Design Suite v 14.7 (Xilinx ${ }^{\circledR}$ Inc., San Jose CA, United States) software, using the Verilog hardware description language. Once the binary programming file was generated by ISE, the design was loaded to the FPGA via an USB port of the PC, using the Mojo Loader v1.3.0 application.

TABLE 1 | Required specifications and features of the FPGA-based architecture of the PG-nFES.

\begin{tabular}{|c|c|}
\hline $\begin{array}{l}\text { Design } \\
\text { specification }\end{array}$ & Description \\
\hline Pulse shape & Rectangular, biphasic \\
\hline $\begin{array}{l}\text { Range of stimulation } \\
\text { parameters }\end{array}$ & $\begin{array}{l}\text { Frequency: } 1-150 \mathrm{~Hz} \text {. } \\
\text { Pulse width: } 20-400 \mu \mathrm{s} . \\
\text { Amplitude: } 1-50 \mathrm{~mA} . \\
\text { Burst time: } 1-4 \mathrm{~s} \text { active (9-6 s rest) } \\
\text { Additional mode: Continuous stimulation }\end{array}$ \\
\hline Control of parameters & Independent, parameter-wise, and channel-wise. \\
\hline Scalability & $\begin{array}{l}\text { Allows extension to a higher number of stimulation } \\
\text { channels }\end{array}$ \\
\hline Safety features & Battery-powered, isolated power supply, emergency stop \\
\hline Interfaces & $\begin{array}{l}\text { Buttons for selection of parameters and functions, Inputs } \\
\text { for triggering stimulation bursts. } \\
\text { Serial interface for communication with controlling devices }\end{array}$ \\
\hline
\end{tabular}




\section{Design and Implementation}

This subsection describes the design of the system, including the overall architecture and signal flow inside the FPGA, and the external circuitry for amplitude control. Also, it describes the settings of the switches for the selection of the operation modes and the active channel. Finally, the inner workings of the pulse generation blocks are explained.

\section{System Architecture}

Figure 1 shows the block diagram of the FPGA-based architecture of the PG-nFES, including:

- Digital interfaces and configuration modules of stimulation options and parameters (Figure 1B, left).

- Modules for building digital pulses and bursts (Figure 1A).

- External circuits to the FPGA, that convert the pairs of monophasic voltage pulses, to biphasic current-controlled pulses (Figure 1B, right).
Below is given a general description of the signal flow within the PG-nFES, with reference to the Figure 1. The main task of the FPGA-based modules is to generate two time-shifted, monophasic pulses with the desired frequency, pulse-width, and burst-time parameters, and one PWM signal containing the amplitude information. Once those signals leave the FPGA, the PWM signal enters the DAC, which in turn generates a DC voltage output signal that modulates the amplitude of the pair of monophasic pulses in the signal mixers. After these conditioning stages, the two monophasic pulses enter the output stage (Leyva et al., 2018), where they are converted to a single, current-controlled, biphasic stimulation pulse with the desired parameters.

Figure 1B highlights (in red) the blocks and signals of the PGnFES which were built and tested in this work (one stimulation channel). The blocks in black dotted lines illustrate the elements of this architecture that need to be replicated to scale up the system from one to $\mathrm{N}$ channels. The next subsections detail the architecture and main functions of the PG-FES.

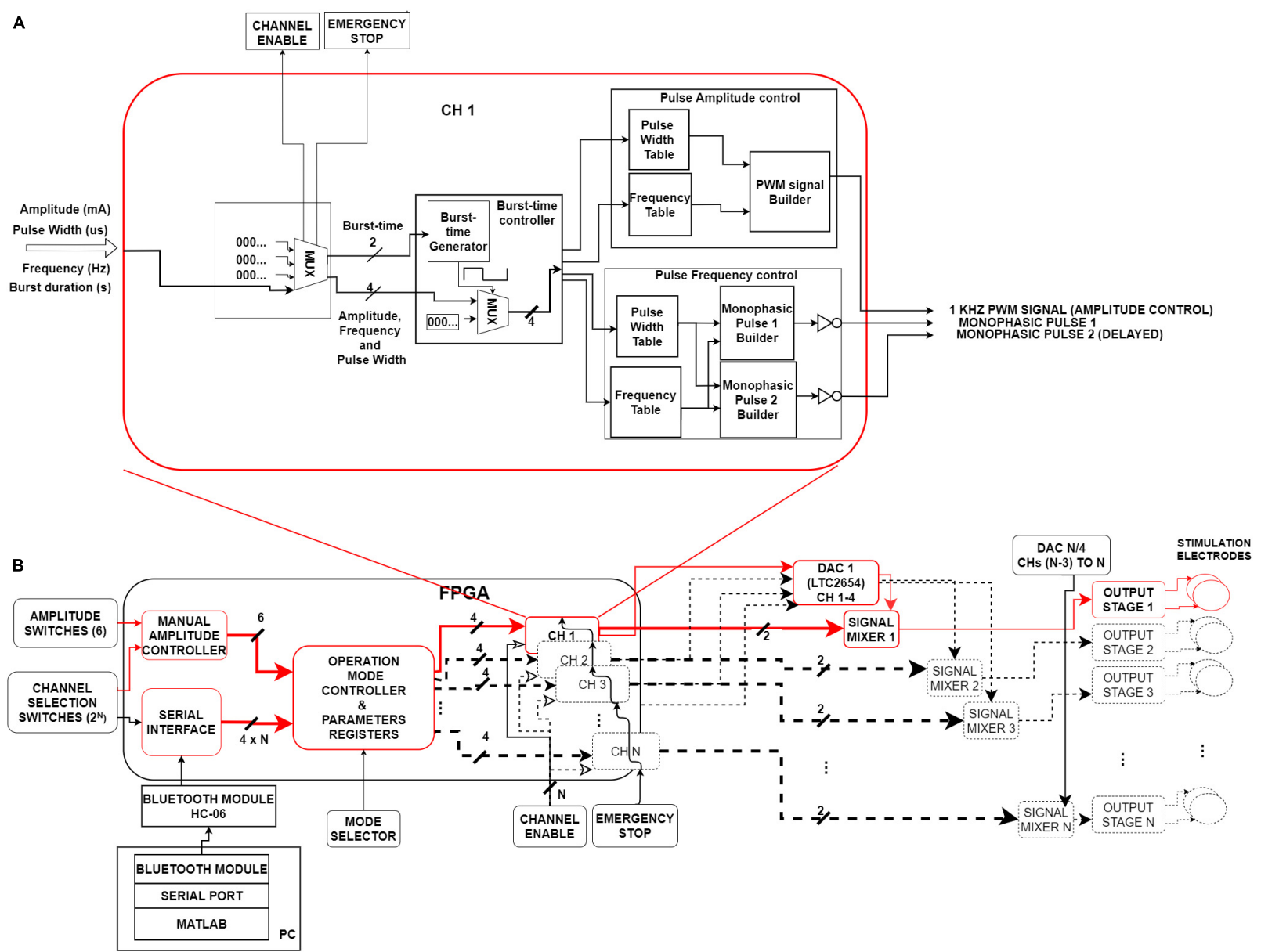

FIGURE 1 | System's architecture. (A) Internal diagram of a single channel $(\mathrm{CH} 1)$ of the PG-nFES. This block forms two time-shifted monophasic pulses (frequency and pulse width), and one PMW signal (amplitude). (B) Left. Digital interfaces and logic blocks for management and storing of stimulation options and parameters, and the main PG-nFES blocks (CH 1 to $\mathrm{CH}$ N). Right. Analog blocks transform the three FPGA outputs to a pair of monophasic pulses with voltage controlled-amplitude, which are combined in the output stage into a single biphasic, current-controlled stimulation pulses. Red blocks and arrows illustrate the implementation of a single FES channel. Blocks with black dotted lines sketch the scaling capability of the architecture from one to $\mathrm{N}$ channels. 


\section{Operation of the Pulse Generator}

The PG-nFES includes several digital inputs and a serial interface, which are used for setting stimulation parameters and operation modes of the system. The functions of these modules are described in Table 2. The following steps describe the procedure to initialize and configure the stimulation parameters of the PG-nFES. The modules involved in this procedure are those illustrated in Figure 1B, unless otherwise noted.

(a) Once an operation mode is selected (Mode Selector switch), the system sets the initial values of the stimulation parameters. For the manual mode, burst time: 3 s ON / 7 s OFF, pulse width: $300 \mu \mathrm{s}$, frequency: $30 \mathrm{~Hz}$, and amplitude: $0 \mathrm{~mA}$. For the serial mode, burst-time: $0 \mathrm{~s}$ OFF (continous stimulation), pulse width: $0 \mu \mathrm{s}$, frequency: $0 \mathrm{~Hz}$, and amplitude: $0 \mathrm{~mA}$.

(b) The Channel Selection Switches define the active channel (n), whose parameters can be set by the external Amplitude

TABLE 2 | Functions of digital inputs and the serial interface of the PG-nFES.

\begin{tabular}{|c|c|c|}
\hline & $\begin{array}{l}\text { Input/function } \\
\text { name (size) }\end{array}$ & Function description \\
\hline \multirow[t]{5}{*}{$\begin{array}{l}\text { Digital } \\
\text { inputs } \\
\text { (Switches) }\end{array}$} & $\begin{array}{l}\text { Channel enable } \\
\text { (N bits, } 1 \\
\text { p/channel) }\end{array}$ & $\begin{array}{l}\text { Switches to enable/disable the stimulation } \\
\text { output of each channel }\end{array}$ \\
\hline & $\begin{array}{l}\text { Channel } \\
\text { selection (N } \\
\text { bits, } 1 \\
\text { p/channel) }\end{array}$ & $\begin{array}{l}\text { Switches to select the channel to be configured } \\
\text { in the manual and serial operation modes. }\end{array}$ \\
\hline & $\begin{array}{l}\text { Amplitude } \\
\text { control (6 bits) }\end{array}$ & $\begin{array}{l}\text { The manual amplitude control comprises six } \\
\text { switches, that codify (in binary) the amplitude } \\
\text { setting, from } 0 \text { (000000) to } 48 \mathrm{~mA}(110000) \text {. }\end{array}$ \\
\hline & $\begin{array}{l}\text { Mode selector } \\
\text { (1 bit) }\end{array}$ & $\begin{array}{l}\text { Switch to change the operation mode of the } \\
\text { PG-nFES: manual or serial }\end{array}$ \\
\hline & $\begin{array}{l}\text { Emergency } \\
\text { stop (1 bit) }\end{array}$ & $\begin{array}{l}\text { It stops or restarts the pulse generation of all } \\
\text { (middle, lower zone of Figure 1B) when its } \\
\text { logic level is set to high or low. When used to } \\
\text { restart stimulation, the last configured } \\
\text { parameters of each channel are maintained }\end{array}$ \\
\hline \multirow[t]{3}{*}{$\begin{array}{l}\text { Serial } \\
\text { interface }\end{array}$} & Serial protocol & $\begin{array}{l}\text { Channel type: Simplex (only receives data from } \\
\text { external devices) } \\
\text { DATA transfer speed: } 9600 \text { bauds (bits per } \\
\text { second) } \\
\text { DATA format: } 1 \text { start bit, } 8 \text { data bits (LSB first), } \\
1 \text { start bit, } 1 \text { stop bit, no parity bit. } \\
\text { Burst-time: The } 3 \text { LSBs codify the } 5 \text { possible } \\
\text { values (0, } 1,2,3 \text { and } 4 \text { s). } \\
\text { Amplitude: The } 6 \text { LSBs codify the } 48 \text { possible } \\
\text { values ( } 1-48 \text { mA. Resolution: } 1 \mathrm{~mA} \text { ) } \\
\text { Frequency: The } 8 \text { bits codify the } 150 \text { possible } \\
\text { values ( } 1-150 \mathrm{~Hz} \text {. Resolution: } 1 \mathrm{~Hz} \text { ) } \\
\text { Pulse-width: The } 6 \text { LSBs codify the } 50 \text { possible } \\
\text { values ( } 10-500 \mu \text { s. Resolution: } 10 \mu \mathrm{s} \text { ) }\end{array}$ \\
\hline & Buffering & $\begin{array}{l}\text { Accumulates } 4 \text { consecutive serial data packets } \\
\text { received ( } 10 \text { bits each), one for each parameter }\end{array}$ \\
\hline & Deserialization & $\begin{array}{l}\text { Upcoming serial data are separated into four } \\
\text { bytes, one for each parameter and sent } \\
\text { separately sent to the Operation Mode } \\
\text { controller (Figure 1B, left side), where } \\
\text { parameter settings are stored in dedicated } \\
\text { registers for each channel }\end{array}$ \\
\hline
\end{tabular}

Switches, or by serial commands received by the HC-06 bluetooth module (Figure 1B, lower left), connected to the internal Serial Interface module inside the FPGA. The configuration and updating of parameters are controlled by the manual amplitude controller or the serial interface module, depending on the operation mode selected.

(c) The Operation Mode Controller module continually reads its inputs and stores the parameter settings in dedicated memory registers for each channel.

(d) Whenever new values are stored in the Parameter Registers module, the outputs of the Operation Mode Controller module are updated for the active channel, while keeping the last settings in all other channels.

\section{Generation of Pulses}

Each $\mathrm{CH} n$ module generates the stimulation pulses and bursts on the $\mathrm{n}$-th channel (Figure 1A). The whole range of values available for each parameter are stored in individual Look Up Tables (LUT) for the burst-time, pulse amplitude and pulse frequency control modules (Figure 1A).

\section{Burst-Time Control Module}

Two intermediate variables, frequency and duty-cycle, are inputs for the Burst-Time Generator module that, in combination with a MUX, control the timing of the burst of pulses. Four values are obtained from the Burst-time Controller module: two for the Pulse amplitude control (frequency and pulse width of the PWM signal) and two for Pulse Frequency Control (pulse width and frequency of the monophasic pulses) modules.

\section{Pulse Frequency Control Module}

This module takes as inputs the outputs of the Pulse Width and Frequency LUTs, and in turn outputs two variables (one for the pulse width parameter and one for the frequency parameter). These variables are used as inputs to each Monophasic Pulse Builder module, where they are used to generate a pair of digital pulses with the value of the pulse width parameter and a $10 \mu \mathrm{s}$ delay between them (repeated at time intervals defined by the frequency variable).

\section{Amplitude Control Module}

The control of amplitude is carried out first in the digital domain, involving the FPGA and the DAC's input, and later in the analog domain, involving the DAC's output, the mixing circuits and the output stage (Figure 1B, right side). This module takes as inputs two intermediate variables coming out from the burst-time controller module: a frequency and a duty cycle of a PWM signal. These two values are associated to LUTs' addresses, that contain the value of counters that define frequency $(1 \mathrm{kHz})$ and the duty cycle (0-98\%) of a PWM signal, the output of this module (Figure 1A).

The output PWM signal is used as input to a 4-channel, 12bit resolution DAC (LTC2645), which translates the PWM signal to a DC voltage $(0-2.5 \mathrm{~V})$ proportional to the duty cycle. The DC voltage is used in the next stage (signal mixer) to modify the amplitude of the pair of monophasic pulses, so that a $0-96 \%$ duty cycle is mapped to $0-48 \mathrm{~mA}$ pulse amplitude. 


\section{Signal Mixer}

This block (Figure 1B, right side) integrates the three stimulation parameters in each monophasic pulse. The signal mixer, comprising mainly an NPN transistor (BC546) configured to operate as an ON/OFF switch, transforms the digital pulses $(3.3 \mathrm{~V})$ to the amplitude set by the DAC's output voltage (0$2.5 \mathrm{~V}$ ) combining the frequency, pulse width and amplitude parameters in each monophasic pulse. Within the signal mixers the signal mixers, the polarity of both pulses is inverted (to normal) before they are sent to the output stage. At the output stage, the two monophasic voltage pulses are combined into a single biphasic, current-controlled pulse, as described in Leyva et al. (2018). A single PG-nFES channel plus the output stage form a complete FES stimulation channel, which underwent technical and application tests as described in the following sections.

\section{Performance Tests of the Functional Electrical Stimulation System}

The performance tests of the FES system were divided in two stages: the technical tests, for verification of the whole range of stimulation parameters settings, and a proof-of-concept, where sequences of electrical stimulation were programmed through the serial interface to produce a set of upper limb movements in healthy participants.

\section{Characterization of Stimulation Parameters}

The manual operation mode was selected to configure a set of electrical stimulation sequences, for characterization of three parameters: amplitude, frequency and pulse width. For each parameter, the one of interest was varied while the other two remain fixed at some typical value. The parameter settings established for the characterization of each parameter (and the incremental steps in their values) are shown in Table 3. It is worth mentioning that the information in Table 3 is different to that of Table 1, which contains the technical specifications required for the device, including the desired values of stimulation parameters.

The pulses delivered at the output stage were applied to a $1 \mathrm{k} \Omega$ load resistor. Five consecutive measurements for each parameter were performed using a current probe (TCP0030A, Tektronix) and a digital oscilloscope (MDO3104, Tektronix) with $1 \mathrm{GHz}$ bandwitdh. For verification and analysis of the data obtained from the measurements, descriptive parametric statistics were calculated for each stimulation parameter:

TABLE 3 | Ranges and steps of stimulation parameters used in the characterization measurements.

\begin{tabular}{lccc}
\hline $\begin{array}{l}\text { Fixed parameters } \\
\text { values }\end{array}$ & $\begin{array}{c}\text { Measured } \\
\text { parameter }\end{array}$ & $\begin{array}{c}\text { Measuring } \\
\text { range }\end{array}$ & Increments \\
\hline $\begin{array}{l}\text { Amplitude: } 16 \mathrm{~mA} \\
\text { Pulse-width: } 300 \mu \mathrm{s}\end{array}$ & Frequency $(\mathrm{Hz})$ & $5-30,30-150$ & 5,10 \\
$\begin{array}{l}\text { Frequency: } 30 \mathrm{~Hz} \\
\text { Amplitude: } 16 \mathrm{~mA}\end{array}$ & $\begin{array}{c}\text { Pulse width } \\
(\mu \mathrm{s})\end{array}$ & $\begin{array}{c}20-50,50-100, \\
100-400\end{array}$ & $10,50,100$ \\
$\begin{array}{l}\text { Frequency: } 30 \mathrm{~Hz} \\
\text { Pulse-width: } 300 \mu \mathrm{s}\end{array}$ & Amplitude $(\mathrm{mA})$ & $1-48$ & 1
\end{tabular}

mean, standard deviation and percentage error (shown in Equations 1-3):

$$
\begin{aligned}
\bar{X} & =\frac{\sum_{i=1}^{N} X_{i}}{N} \\
\sigma & =\sqrt{\frac{\sum_{i}^{N}\left(X_{i}-\bar{X}\right)^{2}}{N}} \\
e & =\frac{\left|V_{\text {Expected }}-V_{\text {Measured }}\right|}{V_{\text {Expected }}} \times 100 \%
\end{aligned}
$$

where $\bar{X}$ is the mean of measurements, $\sigma$ is the standard deviation, $X_{i}$ represents each measurement, $\mathrm{N}$ is the number of measurements, $e$ is the percentage error, $V_{\text {Expected }}$ is the programmed value, and $V_{\text {Measured }}$ is the average of measurements $(\bar{X})$.

Also, for each stimulation parameter the mean absolute percentage error (MAPE), and the coefficient of determination $\left(r^{2}\right)$ were calculated, to assess the overall accuracy and linearity (respectively) of the measurements. Moreover, the repeatability of the characterization measurements for each stimulation parameter was obtained (Portuondo and Portuondo, 2010).

\section{Modulation of Stimulation Parameters. Simulation}

To test the flexibility of the PG-nFES for the control of stimulation parameters, we implemented three patterns of simultaneous modulation of two parameters with the following characteristics:

$$
y 1(t)=\sin (2 \pi f t), f=0.5 \mathrm{~Hz}, t=0.2 s, t_{\max }=8 \mathrm{~s}
$$

(1) Frequency and Pulse Width Modulation:

Frequency range (Hz): $10-90$

Pulse Width range ( $\mu$ s): $20-500$

Amplitude $(\mathrm{mA})=20$ (fixed)

(2) Amplitude and Pulse Width Modulation:

Amplitude range (mA): 1-48

Pulse Width range ( $\mu$ s): $20-500$

Frequency $(\mathrm{Hz})=30$ (fixed)

(3) Frequency and Amplitude Modulation:

Frequency range (Hz): $20-50$

Amplitude range (mA): 1-48 mA

Pulse Width $(\mu \mathrm{s})=100$ (fixed)

The modulation patterns were programmed in MATLAB ${ }^{\circledR}$ scripts and sent via the Bluetooth serial interface of a Laptop PC to the HC-06 receiver connected to the PG-nFES, to update the stimulation parameters as required.

\section{Proof-of Concept: Non-invasive Functional Electrical Stimulation}

A feasibility test of the potential use of the PG-nFES for transcutaneous FES applications was performed using a single FES channel. For this, the PG-nFES coupled to an output 
stage (Leyva et al., 2018) through the DAC and signal mixers (right side of Figure 1B), were used to deliver sequences of electrical stimulation pulses to the arm (through transcutaneous stimulation electrodes) of two able-bodied volunteers, with the aim of producing four different movements of the upper extremity. Before the proof-of-concept session, each participant received, read, and signed an informed consent letter, which was approved by the Research and Ethics Committees of the Institution, based on the principles for research in human beings of the Nuremberg Code and the Helsinki Declaration promulgated by the World Medical Association. In that letter, the participants were informed that some photographs and videos would be recorded during the experimental session, that could be used for scientific and educational purposes, without compromising their identity at any moment.

The test consisted in the application of FES sequences, programmed in the PG-nFES and delivered by the output stage (Figure 1), to generate four upper limb movements: hand opening, power grasp, elbow flexion and arm abduction. For each movement, trains of biphasic pulses were applied through a pair of reusable, self-adhesive surface stimulation electrodes (Durastick Plus ${ }^{\circledast}$, square shaped, $5 \mathrm{~cm} \times 5 \mathrm{~cm}$ ) placed over the forearm and the upper arm. For the hand opening and power grasp movements the electrodes were placed in the forearm, over the motor points for the wrist and hand extensors and flexors muscles. For these movements, an elastic fabric sleeve was placed over the forearm, with marks in suitable positions of stimulation electrodes for these movements (Martín, 2019). For the upper arm movements, the electrodes were placed over the motor points of the biceps brachii for the elbow flexion, and in the lateral deltoid for arm abduction, as recommended in Baker (2000).

A MATLAB $^{\circledR}$ script was written to send wirelessly (via the Bluetooth serial interface of a Laptop PC) serial messages to the HC-06 receiver, which was hardwired to the Serial Interface block of the PG-nFES (Figure 1, lower-left corner). This way, the corresponding serial commands were sent to the PG-nFES, following the data protocol and format explained in Table 2, to set the desired stimulation parameters. The fixed parameters used in FES sequences for all four movements were $30 \mathrm{~Hz}$ frequency, 300 $\mu \mathrm{s}$ pulse-width, and burst-time of $4 \mathrm{~s}$ ON, $6 \mathrm{~s}$ OFF. For each target movement, the stimulation amplitude was increased from $1 \mathrm{~mA}$, in $1 \mathrm{~mA}$ steps, to the level that produced full range of motion without becoming uncomfortable.

\section{Modulation of Parameters. Practical Demonstration}

To test the capacity of our system for modulation of parameters in real conditions, we designed a stimulation pattern comprising simultaneous modulation of frequency and pulse width with a sinusoidal profile (Figure 2). Moreover, the frequency $(20-40 \mathrm{~Hz})$ and pulse width (200-400 $\mu$ s) values were chosen within ranges reported as suitable for FES applications while avoiding rapid muscle fatigue (Downey et al., 2011). Moreover, the combinations of frequency and pulse width were selected to maintain almost constant the charge throughout the duration of each individual FES-induced contraction.

To compare the capabilities of the PG-nFES with conventional FES stimulation (with fixed parameters), two stimulation approaches were used to perform the functional movement "bring a bottle to the mouth": the above-mentioned parameter

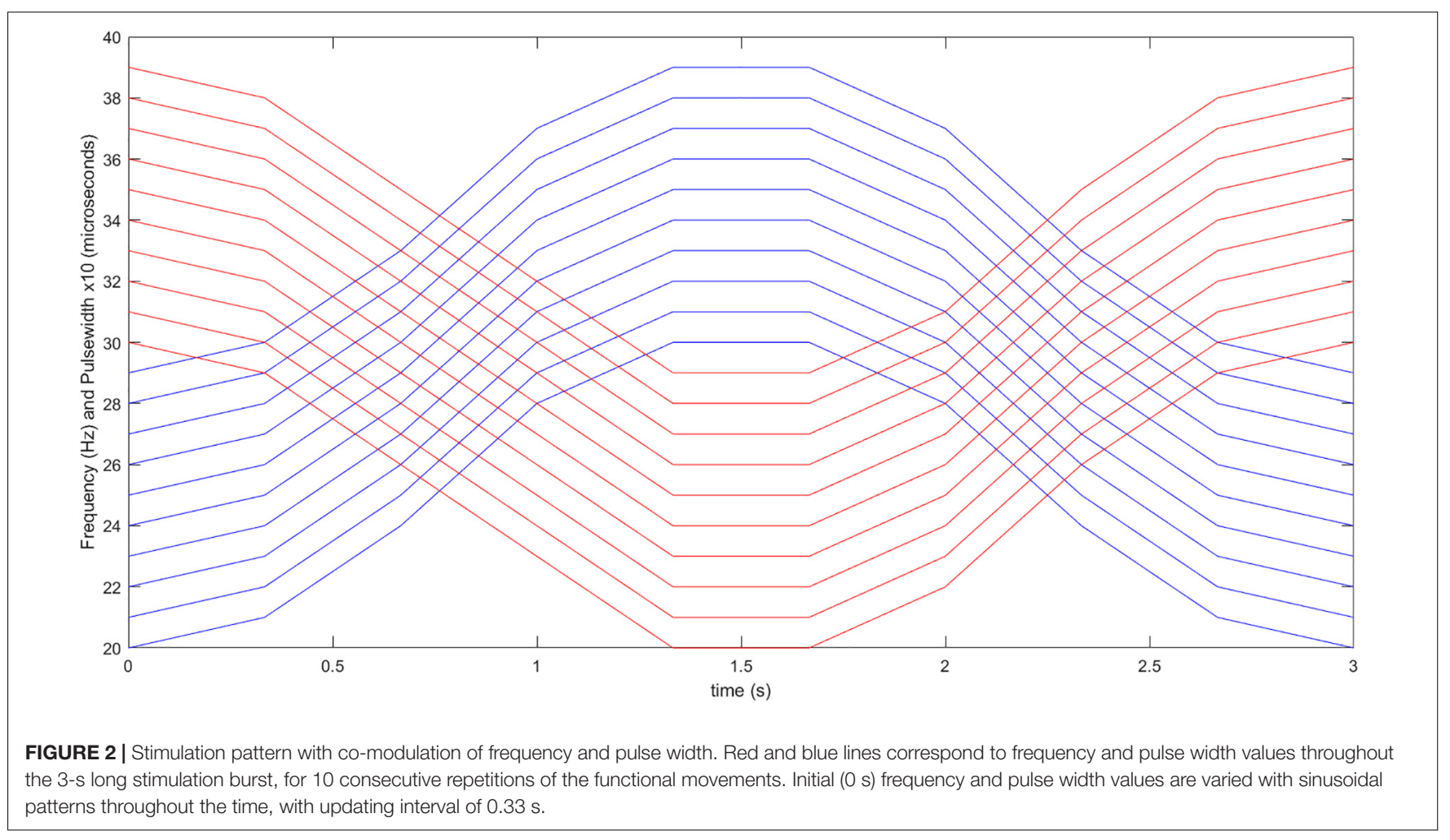


modulation approach, and the conventional FES approach, where constant frequency $(30 \mathrm{~Hz})$ and pulse width $(300 \mu \mathrm{s})$ are employed. To enable this functional movement, a second complete FES stimulation channel was implemented, taking advantage of the scalability features of the PG-nFES, illustrated in Figure 1B. The stimulation pattern with fixed parameters $(30 \mathrm{~Hz} / 300 \mu \mathrm{s})$ was used in the stimulation channel used for the power grasp (finger flexors), and the modulation pattern was used in the stimulation channel used for the elbow flexion (biceps brachii).

Electrical stimulation was applied through pairs of squared $\left(5 \times 5 \mathrm{~cm}\right.$ ) electrodes (Durastick Plus ${ }^{\circledR}$ ) over the midline of the left biceps brachii muscle and over the motor point of the finger flexors in the left forearm of a healthy volunteer. Functional stimulation thresholds (pulse amplitudes) were determined in two conditions: (1) $25 \mathrm{~mA}$ for $30 \mathrm{~Hz} / 300 \mu \mathrm{s}$ pulses (to be used in the fixed parameters condition), and (2) $22 \mathrm{~mA}$ for $40 \mathrm{~Hz} / 400$ $\mu$ s pulses (the highest frequency and pulse width values to be employed in the modulation stimulation pattern). Moreover, the functional threshold for the power grasp movement when using fixed $30 \mathrm{~Hz} / 300 \mu \mathrm{s}$ pulses was $14 \mathrm{~mA}$. Those stimulation amplitudes were kept constant throughout all the stimulation repetitions for both protocols.

Stimulation sequences for each movement repetition were delivered, starting with Channel 1 (power grasp) presented $0.5 \mathrm{~s}$ ahead of Channel 2 (Elbow flexion). The duration of stimulation bursts for Channel 1 was $4 \mathrm{~s}$ while for Channel 2 was $3 \mathrm{~s}$, with a $6 \mathrm{~s}$ rest time after the stimulation in channel 1 ceased $(4 \mathrm{~s})$. This way, there was a $10 \mathrm{~s}$ period between the start of each movement repetition. Twenty repetitions of this stimulation sequence were delivered to the participant's arm, to perform the "bring bottle to mouth" functional movement. A rest time of $20 \mathrm{~min}$ was used between the two protocols, and the participant was blinded to the order of delivery.

During the stimulation session, the subject was sitting in a chair with the front side rotated $90^{\circ}$ relative to the front view angle of the camera. Green squared markers of approximately $1 \mathrm{~cm}^{2}$ were placed in the wrist, elbow, and shoulder joints, to facilitate the extraction of data. In the rest time between stimulation repetitions the bottle of water lied over another chair placed at the side of the participant, while its hand rested over the same chair, with the fingers positioned around the bottle.

\section{Video Recording and Analysis}

The sequence of 20 movement repetitions for each stimulation condition was recorded on video (c922 Pro, Logitech) at 720 $\mathrm{p}$ resolution and 30 frames per second, with the software Logitech Capture (v 2.02.155) running in the same computer used to send the serial commands to the PG-nFES. The video recordings of both stimulation protocols were analyzed with the Kinovea software (v 0.8.15), using the tools to set and follow the markers previously positioned over the bone prominences of the shoulder, elbow and wrist joints, and calculate the angle of the elbow joint throughout the movements. Finally, the minimum elbow joint angle (corresponding to the highest elbow flexion angle) was identified for each movement repetition and protocol. For each protocol, the mean and S.D. of the elbow joint angle measurements were calculated and ploted, and linear regressions were performed.

\section{RESULTS}

\section{Characterization of Stimulation Parameters}

Figure 3 shows the ideal response, the average, standard deviation, and percentage error obtained in the characterization measurements for the three stimulation parameters: amplitude (Figure 3A), frequency (Figure 3B) and pulse width (Figure 3C). From Figure 2, it can be seen that highly linear responses are obtained for the three stimulation parameters with $r^{2}$-values of 0.9998 for the amplitude and pulse width and 1.0000 for the frequency. The corresponding MAPE values were $2.84 \%$ for the amplitude, $2.44 \%$ for the pulse width, and $0 \%$ for the frequency parameter (no errors). The repeatability was 87 and $81.5 \%$ for the amplitude and pulse width, while for the frequency the formula was not suitable (having a MAPE of $0 \%$ ). The statistical quantities obtained from the characterization are concentrated in Table 4 for quick reference.

Noticeable errors (over 5\%) in the measurements were present only for the two smallest amplitude settings (16.4\% and $8.4 \%$, for 1 and $2 \mathrm{~mA}$, respectively) and for the smallest pulse width setting $(16.36 \%$, for $20 \mu \mathrm{s})$. Within the range of parameters commonly used for non-invasive upper limb FES applications (marked in Figure 3 between two green vertical lines for each parameter; 6-35 mA for amplitude, 20-50 Hz for frequency, and 150-400 $\mu s$ for pulse width), the maximum absolute percentage errors were $3.6,0$, and $1.21 \%$, for the amplitude, frequency, and pulse width, respectively.

Figure 4A shows the capture of a biphasic current-controlled pulse, while Figure 4B shows three consecutive bursts of pulses, as they leave the output stage, measured by the current probe of the oscilloscope through the $1 \mathrm{k} \Omega$ load resistor. In Figure $4 \mathrm{~A}$, the desired $300 \mu \mathrm{s}$ pulse width, and $48 \mathrm{~mA}$ peak amplitude of the biphasic stimulation pulse, can be observed. In the middle burst of Figure 4B, the burst-time of $4 \mathrm{~s} \mathrm{ON,} 6 \mathrm{~s}$ OFF, and the peak pulse amplitude of $16 \mathrm{~mA}$ can be appreciated.

\section{Modulation of Stimulation Parameters. Simulation}

Figure 5 shows three different combinations of simultaneous modulation of two (of the three) stimulation parameters. This illustrates the flexibility of the PG-nFES for setting the stimulation parameters. In the lower plots of Figure 5, the persistence of the oscilloscope was increased to enable the visualization of different values of the modulated parameter within a 1-s time window. On the other hand, Figure 6 shows the digital output signals that the PG-nFES generates for four stimulation channels. For each channel the pairs of monophasic pulses can be appreciated, which contain the frequency and pulse width information, and the PWM signals, which convey the amplitude information for each channel. It is worth noting that all channels had different values for the amplitude, frequency 


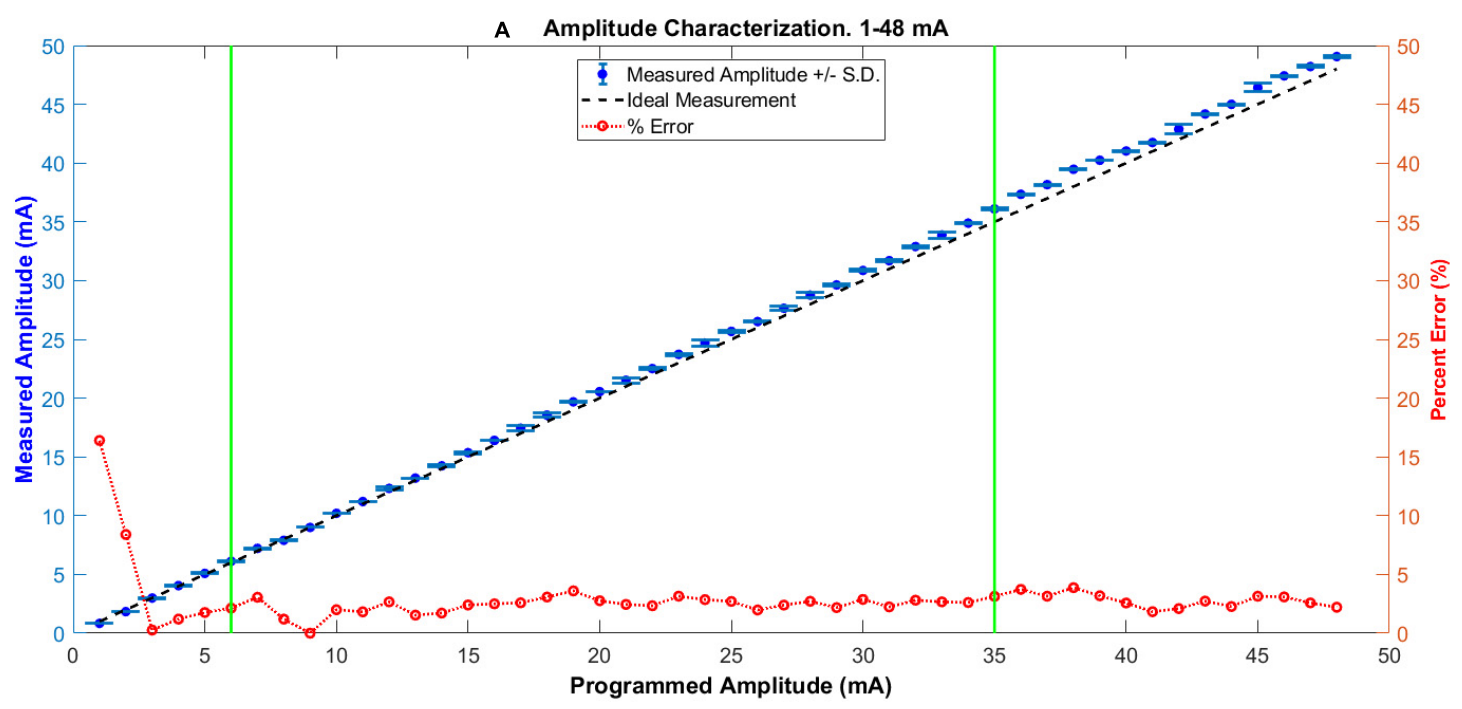

B Frequency Characterization. 5-150 Hz

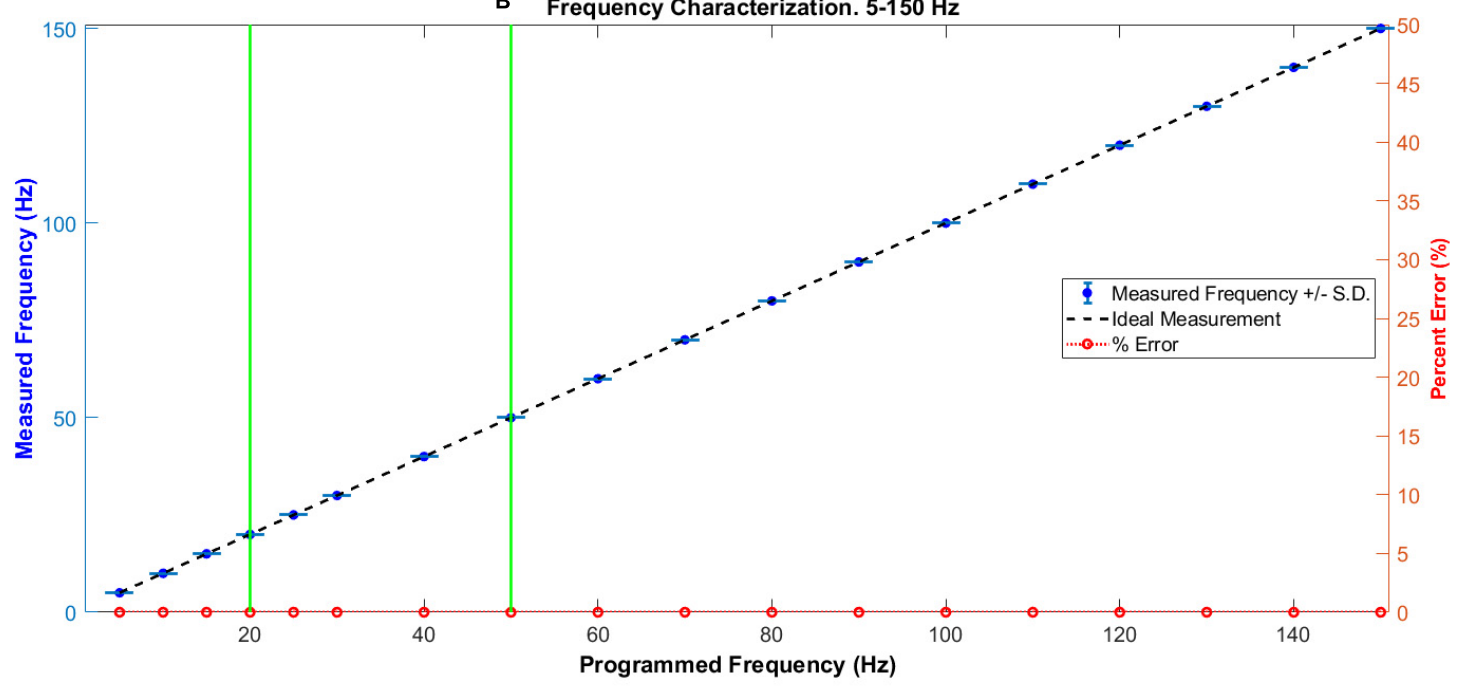

C Pulse Width Characterization. 10-400 $\mu$ s

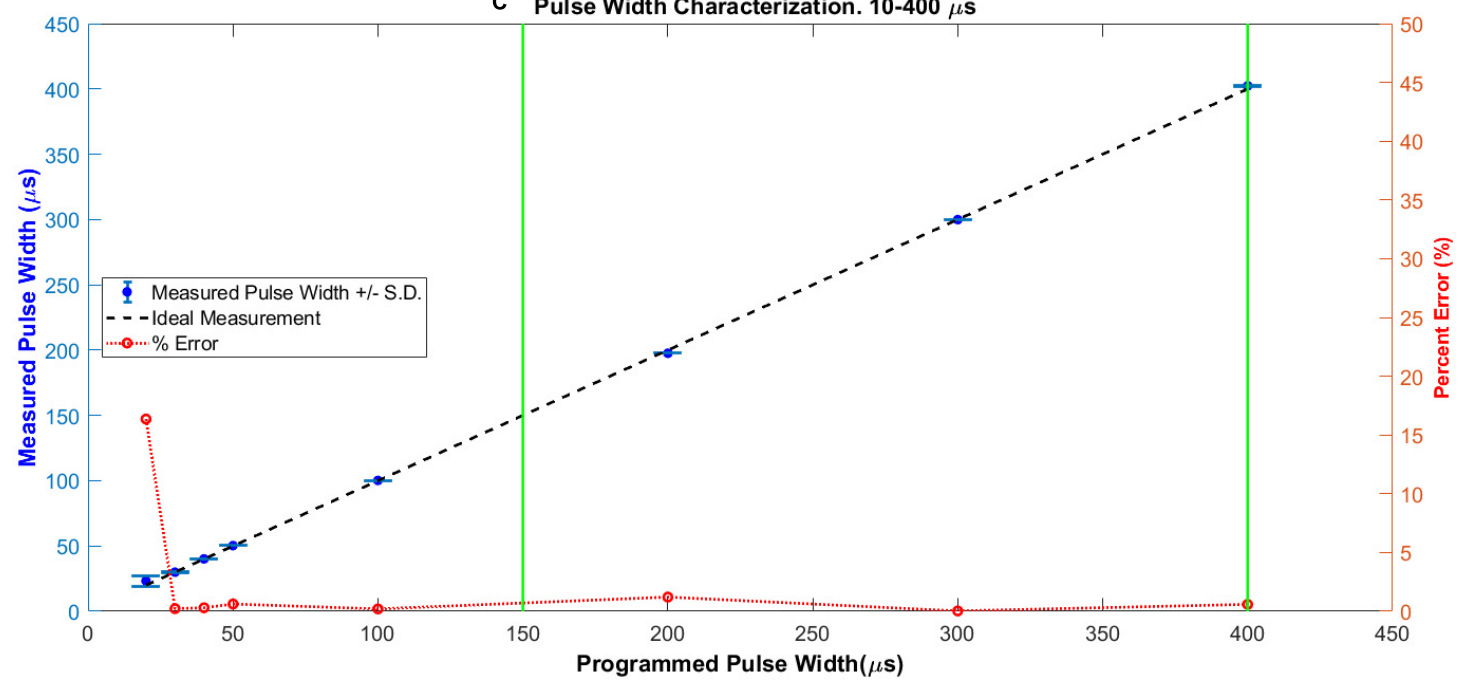

FIGURE 3 | Characterization of stimulation parameters: (A) amplitude, (B) frequency, and (C) pulse width. In each case, the values of parameters among the two vertical green lines define a range commonly used for upper limb FES applications. The maximum percentage error (\%) inside these zones was 3.6, 0, and 1.21, for incises (A-C), respectively. 
TABLE 4 | Results of the statistical quantities obtained from the characterization measurements of the stimulation parameters.

\begin{tabular}{lccc}
\hline & MAPE (\%) & $\boldsymbol{r}^{\mathbf{2}}$ & Repeatability (\%) \\
\hline Amplitude & 2.84 & 0.9998 & 87 \\
Pulse width & 2.44 & 0.9998 & 81.5 \\
Frequency & 0 & 1.0 & 1 \\
\hline
\end{tabular}

and pulse width parameters, and that the corresponding control signals were active at the same time. Although this was not illustrated in Figure 5, the parameters of any channel can be modified without affecting the other parameters of the same or the other channels.

\section{Feasibility Test: Non-invasive Functional Electrical Stimulation}

Figure 7 shows the FES-induced upper limb movements achieved with two healthy volunteers (both males, and 28 years old) by using a single FES stimulation channel. The stimulation parameters and the target muscles over which electrodes were positioned, are presented for the hand opening (Figure 7A), power grasp (Figure 7B), elbow flexion (Figure 7C), and arm abduction (Figure 7D) movements. The four movements were achieved in both subjects (right arm), without any report of discomfort due to the electrodes, the stimulation pulses, or the induced movements.

\section{Modulation of Parameters. Practical Demonstration}

The 20 repetitions of the functional movement "bring the bottle to the mouth" were achieved with each of the two stimulation protocols. Figure 8 shows a screenshot of the video recorded during a single repetition of the movement, showing the positions of the joint markers and an example of the calculation of the elbow joint angle in the Kinovea software. In that picture, we can appreciate the experimental setup including the participant sitting in the chair, the grasped bottle near the mouth and the markers in the corresponding joint bone prominences. Figure 9 summarizes the results of this experiment, where the linear regression equations obtained for both protocols are shown, with slopes of 1.01 and 0.06 , respectively, for the protocols with fixed parameters and co-modulation of frequency and pulse width. Moreover, the average elbow joint angle for both protocols were very similar, with $60.86^{\circ}+/-3.56^{\circ}$ for the modulation pattern, and $61.15^{\circ}+/-7.54^{\circ}$ for the fixed pattern.

\section{DISCUSSION}

In this paper, the functionality of a pulse generator based on an FPGA architecture and its feasibility for non-invasive upper limb FES applications (PG-nFES), were verified through technical characterizations and a proof-of-concept for upper limb FES, when combined with a previously designed output stage (Gutiérrez et al., 2018), that provides biphasic, currentcontrolled electrical stimulation pulses. By setting the PG-nFES with commonly used stimulation parameters for non-invasive neuromuscular applications and surface stimulation electrodes, upper limb movements of the hand and arm (involving the elbow and shoulder joints) were achieved (Figure 7). In contrast, many FPGA-based stimulation systems reported in the literature are targeted to invasive FES applications (Castelli et al., 2017) or are not aimed for upper limb applications (Chen et al., 2009). On the other hand, recent works reporting FES systems for the upper limb (De Marchis et al., 2016; Kutlu et al., 2016; Annetta et al., 2019; Torah et al., 2019; Ward et al., 2020), are designed for custom arrays of stimulation electrodes aimed to small muscles of the forearm to achieve wrist and finger movements. However, these electrode arrays are only available at research settings, which limit their use in the clinics and the adaptation to other FES applications. In contrast, our system is compatible with standard-sized clinical electrodes for transcutaneous stimulation, which can be placed over a variety of body parts and FES applications.

The current level of development of the PG-nFES, according to NASA's TRL (Technology Readiness Levels) scale (Mankins, 2009), is TRL 3 (Experimental critical function and characteristic proof-of-concept). Hence, in this work we focused on the
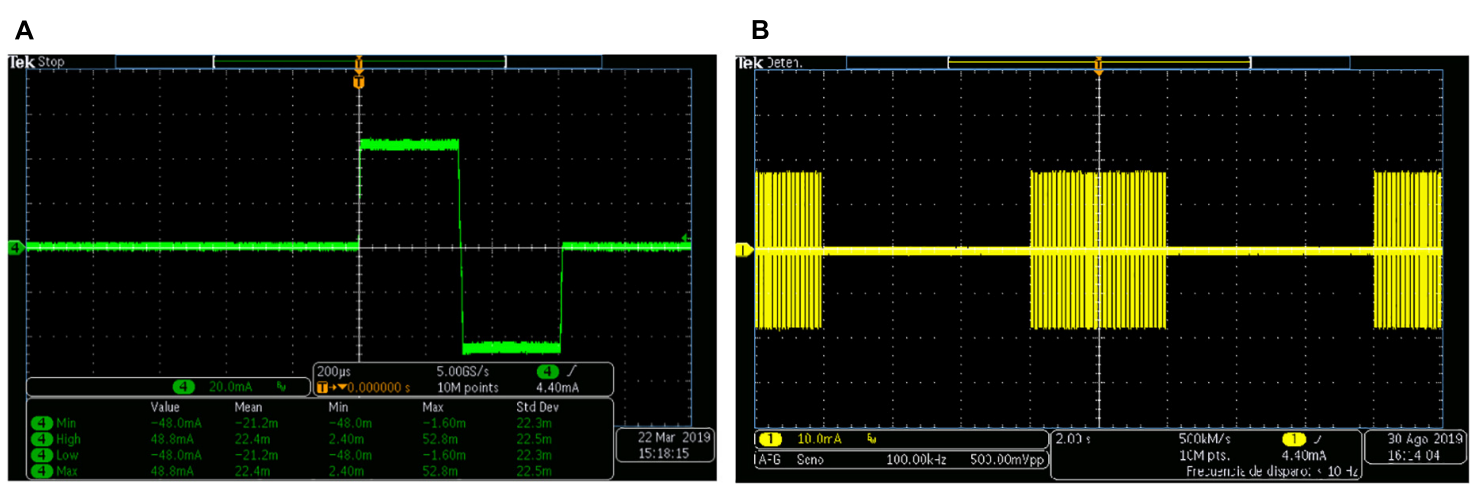

FIGURE 4 | Biphasic stimulation pulses obtained with the PG-nFES. (A) A single stimulation pulse, with $48 \mathrm{~mA}$ amplitude (96 mA peak to peak) and $200 \mu \mathrm{s}$ pulse width. (B) Bursts of stimulation pulses, with burst-time of 4 s ON, 6 s OFF, and peak amplitude of $16 \mathrm{~mA}$. 

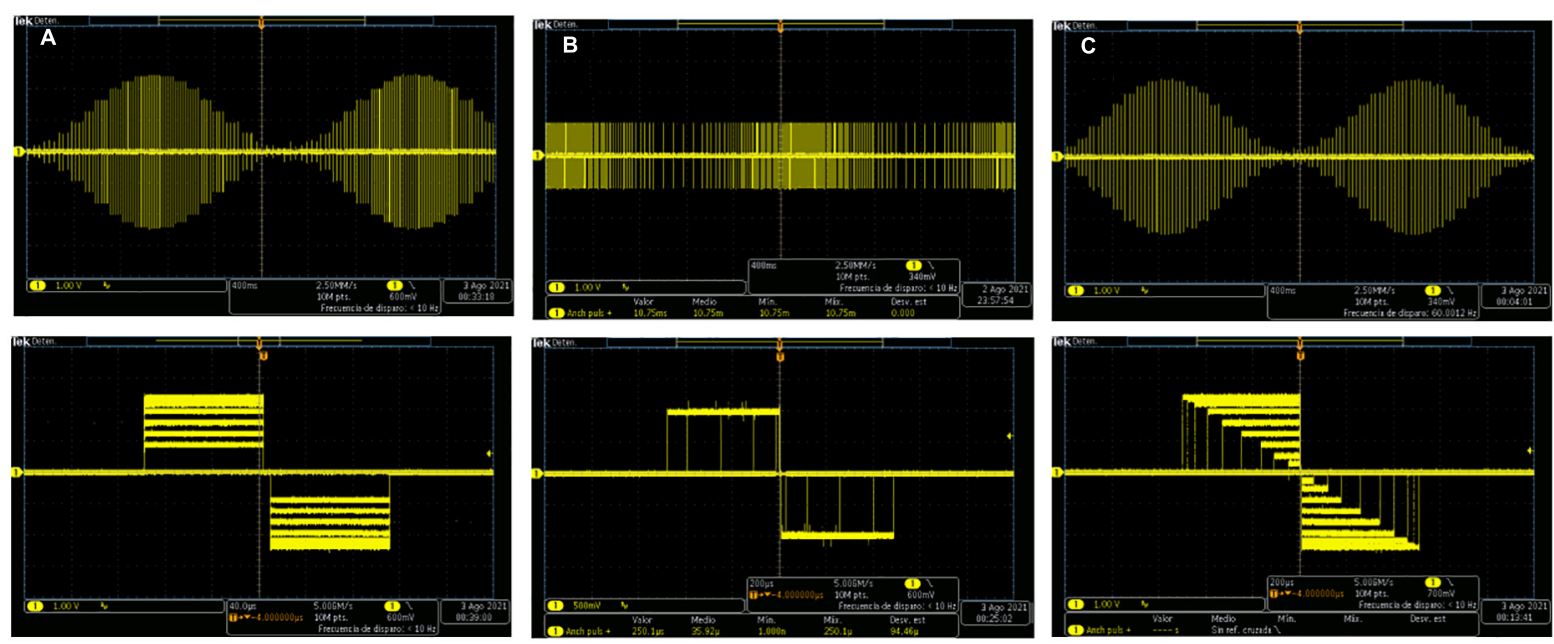

FIGURE 5 | Simultaneous modulation of pairs of two stimulation parameters with the PG-nFES. (A) Frequency and Amplitude Modulation, (B) Frequency and Pulse Width Modulation, (C) Amplitude and Pulse Width Modulation. In the corresponding column for each incise, the upper plot illustrates the slow changing modulation $(0.5 \mathrm{~Hz})$ of one parameter (amplitude or frequency), and the bottom plot shows the modulation of the other parameter (amplitude or pulse width).

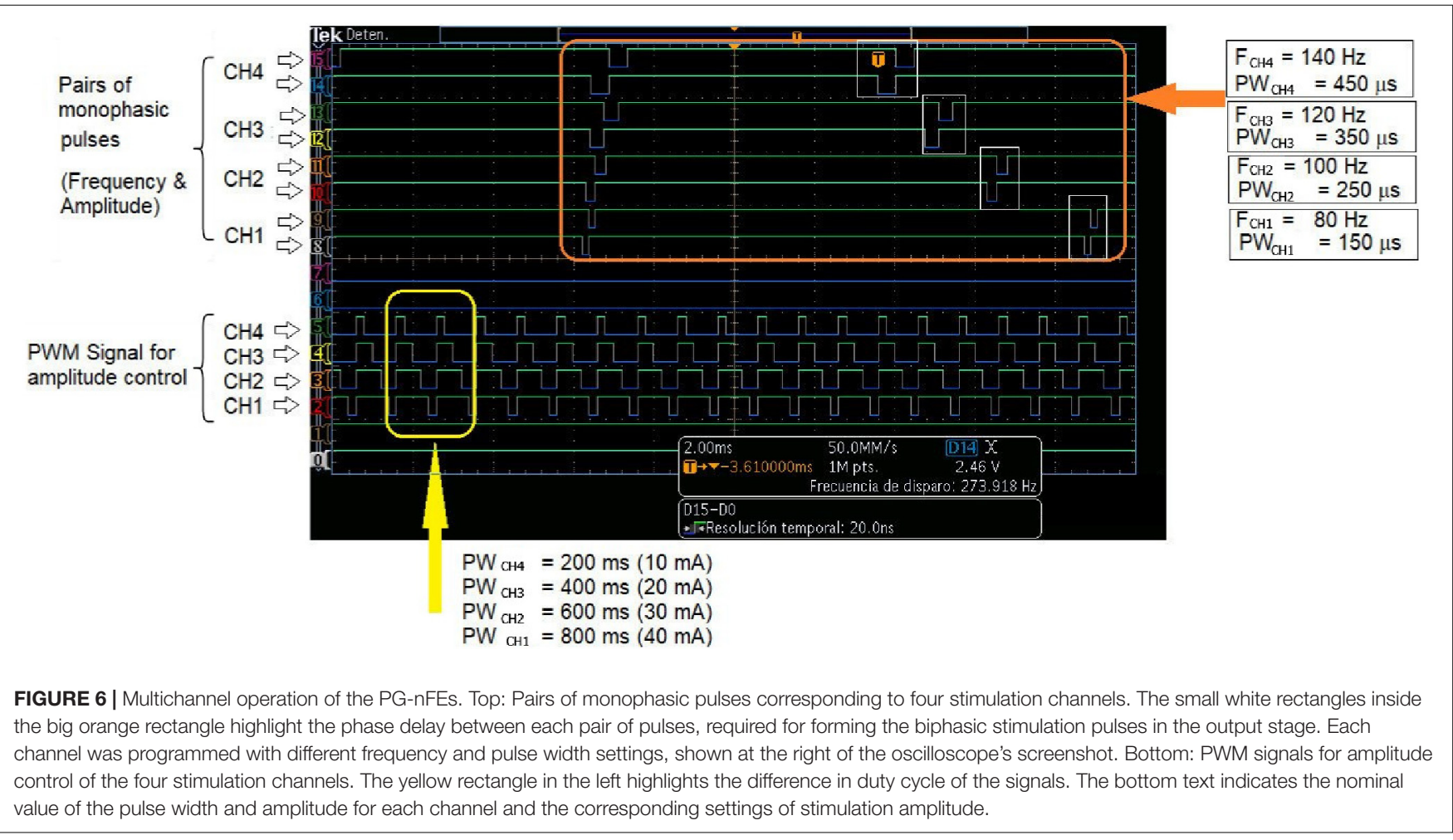

technical validation of the system and performed basic proofsof-concept of non-invasive FES with one and two-channels.

Regarding the characterization of stimulation parameters, it was useful to show the technical feasibility of the system to deliver biphasic stimulation pulses with parameter values within the ranges commonly used in upper limb non-invasive FES applications (Figure 2). This electrical bench test characterization was performed in the required ranges of operation, as needed in an initial proo-of-concept for a technology at the TRL3/TRL4 development level. From the statistical parameters in Table 4, we can see that the system is highly accurate and repeatable in the frequency parameter, and slightly less accurate in the amplitude and pulse width. Regarding linearity, high values were obtained for the three parameters, with an $r^{2}$ coefficient of 0.9998 for both the amplitude and the pulse width, and 1.0 for the frequency (since there were no errors in the measurements). Together, these results support the technical feasibility of the PG-nFES. 


\begin{tabular}{|c|c|c|c|c|}
\hline $\begin{array}{l}\text { Target } \\
\text { movements }\end{array}$ & $\begin{array}{l}\text { Target } \\
\text { muscles }\end{array}$ & \begin{tabular}{|l|}
$\begin{array}{l}\text { Functional } \\
\text { threshold }(\mathrm{mA})\end{array}$ \\
\end{tabular} & Before Stimulation & During Stimulation \\
\hline $\begin{array}{l}\text { Hand } \\
\text { Extension }\end{array}$ & $\begin{array}{l}\text { Finger and } \\
\text { wrist } \\
\text { extensors }\end{array}$ & $\begin{array}{l}\mathrm{s} 1=9 \\
\mathrm{~s} 2=8\end{array}$ & & \\
\hline Power Grasp & $\begin{array}{l}\text { Finger } \\
\text { flexors }\end{array}$ & $\begin{array}{l}s 1=8 \\
s 2=7\end{array}$ & & \\
\hline $\begin{array}{l}\text { Elbow } \\
\text { Flexion }\end{array}$ & $\begin{array}{l}\text { Biceps } \\
\text { Brachii }\end{array}$ & $\begin{array}{l}\mathrm{s} 1=11 \\
\mathrm{~s} 2=14\end{array}$ & & \\
\hline $\begin{array}{l}\text { Arm } \\
\text { abduction }\end{array}$ & $\begin{array}{l}\text { Lateral } \\
\text { Deltoid }\end{array}$ & $\begin{array}{l}\mathrm{s} 1=20 \\
\mathrm{~s} 2=18\end{array}$ & & \\
\hline
\end{tabular}

FIGURE 7 | Proof-of-concept of the PG-nFES for non-invasive transcutaneous FES applications. In the first two columns (from the left), the name of the target movements and muscles are shown. The third column shows the functional thresholds used for each subject to generate each target movement. The fourth and fifth columns contain pictures of the hand or arm before and during (respectively) the delivery of the bursts of stimulation pulses to the target muscles.

The amplitude errors at small values have two possible causes: the minimum measurable value specification of the current probe $(1 \mathrm{~mA})$, or a slight DC offset introduced by the output stage (or a combination of both). Regarding the frequency parameter, the expected value was obtained by the oscilloscope's algorithm for each individual measurement $(0 \%$

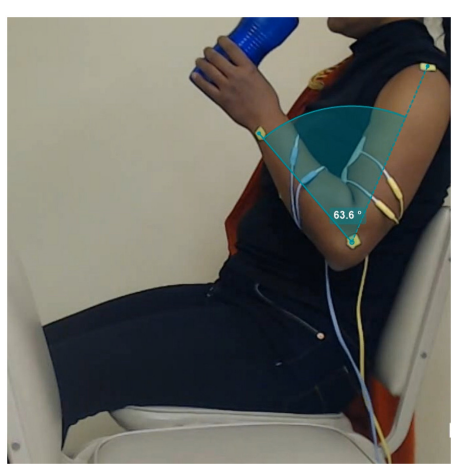

FIGURE 8 | Picture extracted from a frame of video recording of the "bottle to mouth" functional task. The green markers positioned in the shoulder, elbow and wrist joints are used in the Kinovea software to measure the elbow angle for each movement repetition, as illustrated in the green-shaded area. percentage error). For future works, the potential sources of errors will be assessed before adjusting the design and repeating the characterization procedure.

Currently, the maximum selectable amplitude in the PGnFES is $48 \mathrm{~mA}$ peak, which is enough for most non-invasive FES rehabilitation applications for the upper limb. However, the output stage which was combined with the PG-nFES can deliver biphasic, current-controlled pulses over $80 \mathrm{~mA}$ of peak amplitude (Leyva et al., 2018). In the future, an updated version of the output stage will be implemented for a multichannel FES system. Moreover, the use of a 12-bit resolution DAC enables future refinements in the amplitude resolution $(0.5 \mathrm{~mA}$ or less), which has been considered as relevant to improve upper limb FES applications (Schauer, 2017). Furthermore, being the LTC2645 a four-channel DAC based on PWM input signals (without serial interface), the scaling procedure to multichannel systems can be greatly simplified, as illustrated in Figure 6.

When compared to works with similar aims, the FES system based on the PG-nFES can achieve current stimulation pulses of high amplitude (48 mA, suitable for conventional electrodes available at clinics) while allowing total flexibility in the selection for each stimulation parameter and for each stimulation channel. In contrast, other FES systems limit the amplitude to low values (Annetta et al., 2019) or to be the same in all channels 


\section{[Minimum Elbow flexion angle $\left({ }^{\circ}\right)$ for both stimulation approaches}

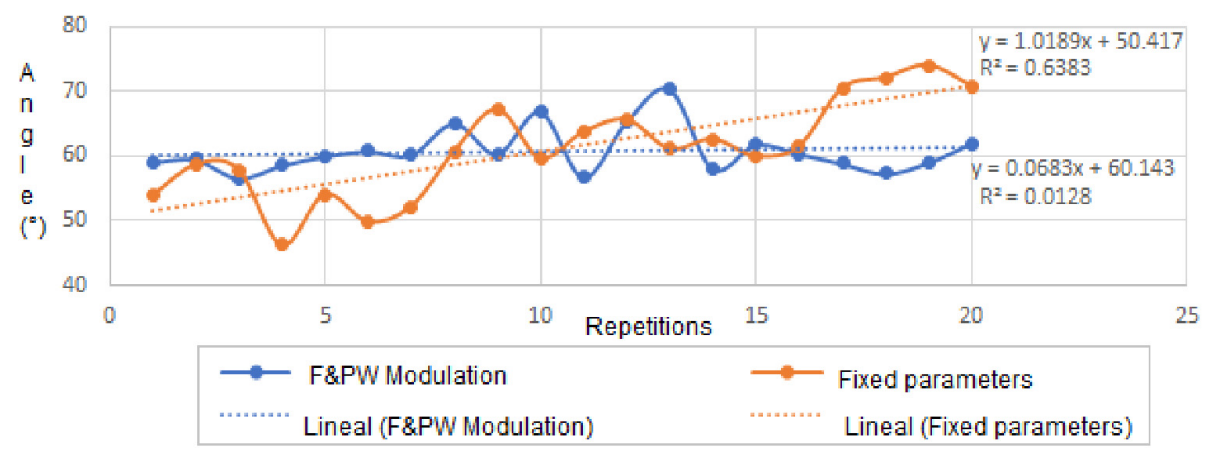

FIGURE 9 | Elbow joint angles for the fixed parameters and co-modulation stimulation protocols throughout the 20 repetitions of the "bottle to mouth" functional task. Linear regression equations and plots for the achieved angles with each stimulation protocol, are shown inside the graph.

(Ward et al., 2020). Regarding the pulse frequency in other FES systems it depends on the number of active channels (Annetta et al., 2019) or is fixed for all of them (Ward et al., 2020). In contrast, our system allows frequencies from 1 to $150 \mathrm{~Hz}$ for each channel and amplitudes from 1 to $48 \mathrm{~mA}$, as shown in Figure 3. Also, multiple channels can be operated and active at the same time, while keeping that flexibility in the selection of stimulation parameters, as shown in Figure 6, where different stimulation amplitudes, frequencies and pulse widths were used for each of four active channels.

In the proof-of-concept the PG-nFES was used to produce movements of different segments of the upper limb (hand, forearm, shoulder). Moreover, a second FES stimulation channel was implemented, taking advantage of the scalability feature of the PG-nFES architecture, while maintaining the flexibility in the configuration of stimulation parameters. To prove further the capabilities of the PG-nFES, the two FES channels were used to perform a clinically relevant functional movement related to a key activity of daily living: "bringing a bottle of water to the mouth." For this, overlapping stimulation sequences for the hand finger flexors and the biceps brachii muscles were required. Different types of stimulation parameters were used for each channel: fixed parameters in one channel (finger flexors) and a pattern of co-modulation of frequency and pulse width in the other channel (biceps). This demonstrated that the scalability and the flexibility of the PG-nFES, since no adjustments to the FPGA firmware were needed.

In the experiment with two stimulation channels, a single participant was included, since the purpose was to show the scalability feature and the flexibility in the configuration of the stimulation parameters. For future developments of the system (TRL 5/TRL 6), further tests will be performed with upper limb movements of the upper limb in unimpaired and impaired subjects (validation in relevant environment). This will be a step forward toward validating the potential of the system for clinical purposes. Also, a complete study of the repeatability of FES-induced movements with the PG-nFES can be performed, comprising several repetitions of different upper limb movements and measurement of electrophysiological and kinematic variables (i.e., sEMG, goniometry, orientation, or acceleration of the limbs). A plausible hypothesis is that, once the stimulation parameters were calibrated for each subject, similar values (before muscle fatigue arises) will be found in those variables across repetitions and/or individuals. Other strategies can be implemented to limit or delay the effects of muscle fatigue, and improve the performance in FES applications, like the pattern of modulation of parameters proposed here and others reported in the literature (Ibitoye et al., 2016).

The complexity of other FPGA-based systems (Annetta et al., 2019) requires that the device in charge of controlling the FES application (PC or embedded) updates constantly the value of each parameter for each single pulse. In our proposed FPGA-based architecture this is not necessary, since the last parameter settings are stored and maintained fixed unless a new parameter update is requested by the application. Also, a pulse generation burst-mode is considered in the PG-nFES, where the active and rest time are chosen as another stimulation parameter, with constant burst stimulation as a special case. Using this feature of the PG-nFES a high flexibility is possible in the parameter settings, since any of them can be updated while the burst-time setting is maintained or adjusted, as required by the application. Examples of the flexible features of the PG-nFES are shown in Figures 5, 6, and especially in Figure 2, where the pattern of co-modulation of frequency and pulse width, for 10 consecutive movement repetitions, is illustrated. Those features were used in the two-channel stimulation sequences for the power grasp and elbow flexion movements, where the burst-mode was used for the power grasp channel (with fixed frequency and pulse width, and amplitude adjusted to the user's personal threshold), while for the elbow flexion channel the continuous stimulation mode with comodulation of frequency and pulse width was employed. It is worth noting that all the configuration and update of the stimulation parameters were performed wirelessly, sending the 
required commands (Table 2) from MATLAB scripts to the FPGA, via a Bluetooth serial interface. This is relevant for safety and portability, since no physical connection to a controlling device is required.

Currently, two of the six available outputs of the PG-nFES have been connected to corresponding DAC channels, signal mixers (amplitude control) and output stages (Figure 1B). Together, they complete two FES stimulation channels. However, within the FPGA all the digital modules for six stimulation channels (Figure 1B, left side) were built and functioning. For each $\mathrm{CHn}$ module, the system can generate simultaneously the three digital signals required: 2 monophasic pulses for frequency and pulse width +1 PWM signal for amplitude control, which were illustrated in Figures 1A, 6. In Figure 6, the scalability feature of the system is illustrated for the case of four channels. The architecture of the PG-nFES was helpful to simplify the scaling of the system to multiple channels, through replication of digital modules inside the FPGA and external analog circuits (Figure 1B). With the implementation of the functional task movement involving two simultaneous movements, each one with different stimulation patterns (fixed and modulated), the potential for a multi-channel version of the system was shown. Such system implementation would allow the use of independent closed-loop controllers for different channels and parameters.

Regarding the modulation pattern, it is relevant to mention that it was designed combining ideas from previous works related to co-modulation of frequency and pulse width for fatigue reduction (Gregory et al., 2007; Chou et al., 2008; Downey et al., 2011), and others suggesting that stimulation frequency should be continually increased during consecutive movement repetitions (Chou et al., 2008). Also, other works were considered that proposed the use of different combinations of pulse width and frequency with equivalent total charge, since they induced constant torque levels (Gregory et al., 2007). The results of the feasibility test of the co-modulation pattern applied to a functional movement, agree with the literature reporting delayed muscle fatigue and improving the performance in FES applications. Future versions of the system would make use of the frequency and pulse width co-modulation approach in patients with impaired CNS conditions that present spasticity, such as spinal cord injury. Using a gradual, variation of the two parameters (Figure 2), while using constant pulse amplitude, can help to reduce the spastic reflex when applying stimulation, by using parameters suggested in the literature for that end (HuiChan and Levin, 1993; Bekhet et al., 2019). This is of main importance since spasticity, as measured by the Ashworth scale, is generally used as an exclusion criterion in clinical FES protocols.

Regarding the results of the functional movement task (Figure 9), the linear regression equations for the elbow joint angle throughout the repetitions (time), have a much lower slope for the fixed parameter pattern. This may indicate a higher stability in the achieved functional movements with the parameter modulation pattern, even when frequency and pulse width were varied during the 3 s-long burst of pulses. Furthermore, the results suggest that the modulation pattern of frequency and pulse width was more effective to maintain the elbow angle more stable (half the S.D) than the fixed pattern, throughout the 20 repetitions. It is worth mentioning that, during the stimulation tests using the modulation protocol the participant reported the biceps contraction felt "stronger toward the end", and the subject was blinded to what type of protocol was being used at any time. This can be further investigated in future works where sEMG electrodes and inertial sensors were used to assess muscle activation and limb movement during the whole experiment. This additional bioelectric and kinematic information can help us to better understand this effects of the stimulation pattern and others.

In the future, the proposed FPGA-based architecture of the PG-nFES could be used to develop a flexible multichannel stimulation system, involving multiple target structures being stimulated in a coordinated manner, as required for noninvasive upper limb FES therapy (Kapadia et al., 2020). When combining such multichannel FES system with a properly designed rehabilitation intervention and clinical study, the potential of this sytem for motor neurorehabilitation could be assessed thoroughly. For this, the technology must be risen from the current TRL3 (proo-of-concept) to at least TRL 6 (prototype tested in relevant environment), according to NASA's classification (Mankins, 2009).

The FPGA-based PG-nFES architecture was designed to allow independent control of stimulation parameters, as shown in the examples of modulation of parameters in Figure 5. Furthermore, this feature was used to implement a stimulation pattern with co-modulation of frequency and pulse width, which was used to deliver stimulation pulses through one stimulation channel to the biceps muscle (elbow flexion), while using a typical FES pattern with fixed frequency and pulse width, in a second channel, for the finger flexors (power grasp). Combining both movements, several repetitions of a functional task could be implemented, as shown in Figures 8, 9. This way we proved the versatility and flexibility of our system.

In the future, the PG-nFES could be used to implement other parameter modulation patterns reported to reduce FESinduced muscle fatigue (Zhou et al., 2016), such as amplitudemodulated pulse-width modulation Furthermore, the system's flexibility in handling the stimulation parameters, can be exploited to implement FES applications based on spatially and time distributed stimulation strategies, which promise to reduce muscle fatigue (Nguyen et al., 2011; Maneski et al., 2013) and increase force (Buckmire et al., 2018).

We identify two main contributions of the PG-nFES to the design of FES systems, to mention:

(1) The architecture of the PG-nFES (Figure 1) enables easy scaling to multiple stimulation channels, since additional logic blocks can be configured in the FPGA to replicate the control logic for the extra channels. This was illustrated schematically in Figures 1, and in Figure 6 with digital signals for the control of 4 channels at the output of the PGnFEs. Finally, the scalability feature it was demonstrated with the implementation of two complete FES channels, and their application in a functional task involving finger and elbow movements (Figure 8). 
(2) The PG-nFEs allows high flexibility in the selection of stimulation parameters, even in the multichannel case. Again, this feature was illustrated in Figures 1A, 6, and demonstrated in practice with the functional task "bringing the bottle of water to the mouth," where two different stimulation patterns were used for each stimulation channel: fixed and modulated frequency and pulse width.

These features are possible, in part, by using Lookup Tables with all the timer values required to handle the four stimulation parameters (burst-time, amplitude, frequency, and pulse width) in their whole operational ranges, and individual registers for each stimulation parameter and channel (Figure 1A). Moreover, a simple PWM module inside the FPGA is implemented to control the pulse amplitude for each stimulation channel (combined with the DAC with PWM inputs and the "signal mixer" blocks). In contrast, most electrical stimulation designs (either based on microcontrollers or FPGAs) use serially controlled (SPI or I2C) DACs to build the pulse shape, width, and amplitude, completely within the DAC (Nogueira et al., 2017). However, it is simpler and more efficient to implement one PWM module inside the FPGA for each stimulation channel, than to implement an SPI communication module for handling as many DACs as stimulation channels.

It is important to emphasize that, besides the technical features of the system mentioned above, biphasic rectangular shaped stimulation pulses, with constant current, are used, which are preferred for safe and efficient non-invasive FES stimulation. In contrast, other FPGA-based designs simplify the pulse shape (Annetta et al., 2019), or keep fixed the value of frequency or amplitude parameters in all channels (Ward et al., 2020), to enable multichannel operation.

Finally, the capabilities of the PG-nFES enable the development of highly customized electrical stimulation sequences for a variety of users and applications. Hence, this architecture can be used as base for the design of robust devices for FES and other electrical stimulation modalities where flexible selection of stimulation parameters in multiple channels might be relevant. Such is the case of brain or spinal cord stimulation, for neuromodulation and rehabilitation applications. In our case, the next step is to integrate the PG-nFES as a component of a multichannel FES-based neuroprosthesis, controlled by a BrainComputer Interface based on evoked potentials, which can be

\section{REFERENCES}

Ambroise, M., Levi, T., Joucla, S., Yvert, B., and Saïghi, S. (2013). Real-time biomimetic central pattern generators in an FPGA for hybrid experiments. Front. Neurosci. 7:215. doi: 10.3389/fnins.2013.00215

Amerijckx, C., Doguet, P., Mével, H., Legat, J. D., Troosters, M., Trullemans, C., et al. (1998). "An electronic device for nerve stimulation," in Proceedings of the Workshop on Industrial Microtechnology Applications, October 15-16, 1998, Madrid.

Annetta, N. V., Friend, J., Schimmoeller, A., Buck, V. S., Friedenberg, D. A., Bouton, C. E., et al. (2019). A high definition noninvasive neuromuscular electrical stimulation system for cortical control of combinatorial rotary hand used for upper limb motor rehabilitation of stroke and spinal cord injury patients.

\section{DATA AVAILABILITY STATEMENT}

The datasets presented in this article are not readily available due to Institutional Policies. Requests to access the datasets should be directed to the corresponding author.

\section{AUTHOR CONTRIBUTIONS}

JG-M: project conception and evaluation of the proof of concept, writing, review, and acceptance of the manuscript. JM-G: design and implementation of the pulse generator, FES channels, characterizations and proof-of-concept, data acquisition and analysis, writing, review, and acceptance of the manuscript. RD: implementation and experimental tests of the pulse generator, review, and acceptance of the manuscript. IH-P: output stage design and tests with the pulse generator, review, and acceptance of the manuscript. JQ-F, AV-H, and LL-S: data interpretation, writing, review, and acceptance of the manuscript. All authors contributed to the article and approved the submitted version.

\section{FUNDING}

This work was supported by the National Council of Science and Technology of Mexico (CONACYT) through the grant SALUD2016-1-272983 and by the projects CYTED-DITECROD218RT0545 and Proyecto IV-8, Amexcid-AUCI.

\section{ACKNOWLEDGMENTS}

We acknowledge the manuscript writing training team (CEMAI in Spanish) of CONACYT, for their valuable help with reviews and constructive criticism of an early version of the manuscript. A special acknowledgment to Desirée López Palafox as an external assessor, and to Beatriz Sanabria, Luis Flores, and Ian Perrilliat, Biomedical Engineering students, for their assistance in building and testing preliminary versions of different blocks of the system.

movements in a human with tetraplegia. IEEE Trans. Biomed. Eng. 66, 910-919. doi: 10.1109/TBME.2018.2864104

Arabi, K., and Sawan, M. A. (1999). Electronic design of a multichannel programmable implant for neuromuscular electrical stimulation. IEEE Trans. Rehabil. Eng. 7, 204-214. doi: 10.1109/86.769411

Baker, L. (2000). Neuromuscular Electrical Stimulation: A Practical Guide, 4th Edn. Downey, CA: Los Amigos Research \& Education Institute.

Bekhet, A. H., Bochkezanian, V., Saab, I. M., and Gorgey, A. S. (2019). The effects of electrical stimulation parameters in managing spasticity after spinal cord injury: a systematic review. Am. J. Phys. Med. Rehabil. 98, 484-499.

Benzaba, W., Allam, A., Bendaouia, L., Boutaleb, R., Ykhlef, F., and Boucetta, Y. (2008). "Anti pain stimulator using FPGA circuit," in Proceedings of the 
2008 2nd International Conference on Signals, Circuits and Systems (Monastir). doi: 10.1109/ICSCS.2008.4746867

Buckmire, A. J., Lockwood, D. R., Doane, C. J., and Fuglevand, A. J. (2018). Distributed stimulation increases force elicited with functional electrical stimulation. J. Neural Eng. 15:026001. doi: 10.1088/1741-2552/AA9820

Castelli, J., Kolbl, F., Siu, R., N'Kaoua, G., Bornat, Y., Mangalore, A., et al. (2017). "An IC-based controllable stimulator for respiratory muscle stimulation investigations," in Proceedings of the Annual International Conference of the IEEE Engineering in Medicine and Biology Society (Piscataway, NJ: Institute of Electrical and Electronics Engineers Inc.), 1970-1973. doi: 10.1109/EMBC. 2017.8037236

Chen, K., Chen, T. C., Cockerham, K., and Liu, W. (2009). "Closed-loop eyelid reanimation system with real-time blink detection and electrochemical stimulation for facial nerve paralysis," in Proceedings of the IEEE International Symposium on Circuits and Systems (Taipei), 549-552. doi: 10.1109/ISCAS. 2009.5117807

Chou, L.-W., Lee, S. C., Johnston, T. E., and Binder-Macleod, S. A. (2008). The effectiveness of progressively increasing stimulation frequency and intensity to maintain paralyzed muscle force during repetitive activation in persons with spinal cord injury. Arch. Phys. Med. Rehabil. 89, 856-864.

Chung, W.-Y., Chuang, C.-C., Liao, Y.-L., and Chang, C.-W. (2004). A new microstimulator with pulse width modulation. J. Med. Biol. Eng. 24, 125-131.

De Marchis, C., Monteiro, T. S., Simon-Martinez, C., Conforto, S., and Gharabaghi, A. (2016). Multi-contact functional electrical stimulation for hand opening: electrophysiologically driven identification of the optimal stimulation site. J. NeuroEng. Rehabil. 13:22. doi: 10.1186/s12984-016-0129-6

Downey, R. J., Bellman, M., Sharma, N., Wang, Q., Gregory, C. M., and Dixon, W. E. (2011). A novel modulation strategy to increase stimulation duration in neuromuscular electrical stimulation. Muscle Nerve 44, 382-387.

Eraifej, J., Clark, W., France, B., Desando, S., and Moore, D. (2017). Effectiveness of upper limb functional electrical stimulation after stroke for the improvement of activities of daily living and motor function: a systematic review and metaanalysis. Syst. Rev. 6, 1-21. doi: 10.1186/s13643-017-0435-5

Gregory, C. M., Dixon, W., and Bickel, C. S. (2007). Impact of varying pulse frequency and duration on muscle torque production and fatigue. Muscle Nerve $35,504-509$.

Grimm, F., and Gharabaghi, A. (2016). Closed-loop neuroprosthesis for reach-tograsp assistance: combining adaptive multi-channel neuromuscular stimulation with a multi-joint arm exoskeleton. Front. Neurosci. 10:284. doi: 10.3389/fnins. 2016.00284

Gutiérrez, A. E. L., Martínez-Méndez, R., and Serrano, P. D. A. (2018). ReCIBE. Rev. Electrón. Comput. Inf. Bioméd. Electrón. 7, 117-135.

Hsueh, Y. H., Yin, C., and Chen, Y. H. (2015). Hardware system for real-time EMG signal acquisition and separation processing during electrical stimulation. J. Med. Syst. 39, 1-8. doi: 10.1007/s10916-015-0267-6

Hui-Chan, C. W., and Levin, M. F. (1993). Stretch reflex latencies in spastic hemiparetic subjects are prolonged after transcutaneous electrical nerve stimulation. Can. J. Neurol. Sci. 20, 97-106.

Ibitoye, M. O., Hamzaid, N. A., Hasnan, N., Khairi, A., Wahab, A., and Davis, G. M. (2016). Strategies for rapid muscle fatigue reduction during FES exercise in individuals with spinal cord injury: a systematic review. PLoS One 11:e0149024. doi: 10.1371/journal.pone.0149024

Jovičić, N. S., Saranovac, L. V., and Popović, D. B. (2012). Wireless distributed functional electrical stimulation system. J. Neuroeng. Rehabil. 9:54. doi: 10.1186/ 1743-0003-9-54

Kapadia, N., Moineau, B., and Popovic, M. R. (2020). Functional electrical stimulation therapy for retraining reaching and grasping after spinal cord injury and stroke. Front. Neurosci. 14:718. doi: 10.3389/fnins.2020.00718

Karami, E., Ahmadi, A., Ahmadi, M., and Saif, M. (2016). "Hardware implementation of deep brain stimulator on a biophysical neural population model," in Proceedings of the International Joint Conference on Neural Networks, 2016-October (Vancouver, BC), 4695-4700. doi: 10.1109/IJCNN.2016.7727816

Kesar, T., Chou, L.-W., and Binder-Macleod, S. A. (2008). Effects of stimulation frequency versus pulse duration modulation on muscle fatigue. J. Electromyogr. Kinesiol. 18, 662-671. doi: 10.1016/j.jelekin.2007.01.001

Kutlu, M., Freeman, C. T., Hallewell, E., Hughes, A. M., and Laila, D. S. (2016). Upper-limb stroke rehabilitation using electrode-array based functional electrical stimulation with sensing and control innovations. Med. Eng. Phys. 38, 366-379. doi: 10.1016/j.medengphy.2016.01.004

Kutlu, M., Freeman, C., Hughes, A.-M., and Spraggs, M. (2017). A home-based FES System for upper-limb stroke rehabilitation with iterative learning control. IFAC PapersOnLine 50, 12089-12094. doi: 10.1016/J.IFACOL.2017.08.2153

Leyva, A., Hernández, I., Martínez-Menendez, R., and Alonso, P. (2018). Output stage of a transcutaneous stimulator for functional electrical and transcutaneous electrical spinal-cord stimulation. Rev. Electrón. Comput. Inf. Bioméd. Electrón. 7, 117-135.

Luzio de Melo, P., da Silva, M. T., Martins, J., and Newman, D. (2015). A microcontroller platform for the rapid prototyping of functional electrical stimulation-based gait neuroprostheses. Artif. Organs 39, E56-E66. doi: 10. 1111/aor. 12400

Maneski, L. Z. P., Malešević, N. M., Savić, A. M., Keller, T., and Popović, D. B. (2013). Surface-distributed low-frequency asynchronous stimulation delays fatigue of stimulated muscles. Muscle Nerve 48, 930-937. doi: 10.1002/mus. 23840

Mankins, J. C. (2009). Technology readiness assessments: a retrospective. Acta Astronaut. 65, 1216-1223. doi: 10.1016/j.actaastro.2009.03.058

Martín, A. I. (2019). Manga Para el Posicionamiento Estratégico de Electrodos de Registro de sEMG y de Estimulación Eléctrica Funcional en Miembro Superior Para su Uso en Una Neuroprótesis [Bachelor's thesis]. Mexico City: Universidad La Salle.

Nekoukar, V. (2021). Control of functional electrical stimulation systems using simultaneous pulse width, amplitude, and frequency modulations. Neuromodulation 24, 1467-1474. doi: 10.1111/NER.13126

Nguyen, R., Masani, K., Micera, S., Morari, M., and Popovic, M. R. (2011). Spatially distributed sequential stimulation reduces fatigue in paralyzed triceps surae muscles: a case study. Artif. Organs 35, 1174-1180. doi: 10.1111/j.1525-1594. 2010.01195.x

Nogueira, R. R., Souza, D. C., Palma, J. C., Nogueira-Neto, G. N., and Nohama, P. (2017). The output circuit of a biphasic constant current electrical stimulator. IFMBE Proc. 60, 621-625. doi: 10.1007/978-981-10-4086-3_156

Osuagwu, B. C. A., Wallace, L., Fraser, M., and Vuckovic, A. (2016). Rehabilitation of hand in subacute tetraplegic patients based on brain computer interface and functional electrical stimulation: a randomised pilot study. J. Neural Eng. 13:065002. doi: 10.1088/1741-2560/13/6/065002

Popovic, M. R., Keller, T., Pappas, I. P. I., and Müller, P. Y. (2001). "Compex motion - new portable transcutaneous stimulator for neuroprosthetic applications," in Proceedings of the 2001 European Control Conference (Porto), 3945-3950. doi: 10.23919/ecc.2001.7076551

Popovic, M. R., Thrasher, T. A., Zivanovic, V., Takaki, J., and Hajek, V. (2005). Neuroprosthesis for retraining reaching and grasping functions in severe hemiplegic patients. Neuromodulation 8, 58-72. doi: 10.1111/j.1094-7159.2005. 05221.x

Portuondo, Y., and Portuondo, J. (2010). La repetibilidad y la reproducibilidad en el aseguramiento de la calidad de los procesos de medición. Tecnol. Química $117-121$.

Quandt, F., and Hummel, F. C. (2014). The influence of functional electrical stimulation on hand motor recovery in stroke patients: a review. Exp. Transl. Stroke Med. 6:9. doi: 10.1186/2040-7378-6-9

Ranganathan, V., Nakahara, J., Samejima, S., Tolley, N., Khorasani, A., Moritz, C. T., et al. (2019). "NeuralCLIP: a modular FPGA-based neural interface for closed-loop operation," in Proceedings of the 9th International IEEE/EMBS Conference on Neural Engineering, NER, 2019-March (San Francisco, CA), 791-794. doi: 10.1109/NER.2019.8717135

Ruiz-Rosero, J., Ramirez-Gonzalez, G., and Khanna, R. (2019). Field programmable gate array applications-a scientometric review. Computation 7:63. doi: 10.3390/COMPUTATION7040063

Sawan, M., Robin, S., Provost, B., Eid, Y., and Arabi, K. (1996). Wireless implantable electrical stimulator based on two FPGAs. Proc. IEEE Int. Conf. Electron. Circuits Syst. 2, 1092-1095. doi: 10.1109/icecs.1996.584611

Schauer, T. (2017). Sensing motion and muscle activity for feedback control of functional electrical stimulation: ten years of experience in Berlin. Annu. Rev. Control 44, 355-374. doi: 10.1016/J.ARCONTROL.2017.09.014

Shahdoost, S., Nudo, R. J., and Mohseni, P. (2017). Generation of stimulus triggering from intracortical spike activity for brain-machine-body interfaces 
(BMBIs). IEEE Trans. Neural Syst. Rehabil. Eng. 25, 998-1008. doi: 10.1109/ TNSRE.2016.2615270

Simpson, J., and Ghovanloo, M. (2007). "An experimental study of voltage, current, and charge controlled stimulation front-end circuitry," in Proceedings of the 2007 IEEE International Symposium on Circuits and Systems (New Orleans, LA), 325-328. doi: 10.1109/ISCAS.2007. 378401

Stewart, A. M., Pretty, C. G., and Chen, X. (2016). "Design and testing of a novel, low-cost, low-voltage, functional electrical stimulator," in Proceedings of the 2016 12th IEEE/ASME International Conference on Mechatronic and Embedded Systems and Applications (MESA) (Auckland), 1-6. doi: 10.1109/MESA.2016. 7587155

Sugiura, T., Khan, A. U., Yu, J., Takeuchi, Y., Kameda, S., Kamata, T., et al. (2016). "A programmable controller for spatio-temporal pattern stimulation of cortical visual prosthesis," in Proceedings of the 2016 IEEE Biomedical Circuits and Systems Conference, BioCAS (Shanghai), 432-435. doi: 10.1109/BioCAS.2016. 7833824

Thanasoulis, V., Partzsch, J., Hartmann, S., Mayr, C., and Schuffny, R. (2012). "Dedicated FPGA communication architecture and design for a large-scale neuromorphic system," in Proceedings of the 2012 19th IEEE International Conference on Electronics, Circuits, and Systems, ICECS (Seville), 877-880. doi: 10.1109/ICECS.2012.6463548

Thrasher, A., Graham, G. M., and Popovic, M. R. (2005). Reducing muscle fatigue due to functional electrical stimulation using random modulation of stimulation parameters. Artif. Organs 29, 453-458. doi: 10.1111/j.1525-1594. 2005.29076.x

Torah, R., Beeby, S., Kostic, M., Keller, T., Lanceros-Méndez, S., Ersman, P. A., et al. (2019). Introduction to EU-H2020 Project WEARPLEX: Wearable Multiplexed Biomedical Electrodes. Available online at: https://eprints.soton.ac.uk/ 435741/1/WEARPLEX_LOPEC_presentation_vFinal.pdf (accessed March 21, 2019).

Venugopalan, L., Taylor, P. N., Cobb, J. E., and Swain, I. D. (2015). Upper limb functional electrical stimulation devices and their man-machine interfaces. J. Med. Eng. Technol. 39, 471-479. doi: 10.3109/03091902.2015. 1102344

Vodovnik, L., Crochetiere, W. J., and Reswickw, J. B. (1967). Control of a skeletal joint by electrical stimulation of antagonists. Med. Biol. Eng. 5, 97-109. doi: 10.1007/BF02474498
Ward, T., Graham, N., Freeman, C., Wei, Y., Hughes, A.-M., Power, C., et al. (2020). Multichannel biphasic muscle stimulation system for post stroke rehabilitation. Electronics 9:1156. doi: 10.3390/electronics9071156

Wong, Y. T., Suaning, G. J., Dokos, S., Preston, P. J., Dommel, N., Grace, D., et al. (2005). "An FPGA-based vision prosthesis prototype: Implementing an efficient multiplexing method for addressing electrodes," in Proceedings of the 27th Annual International Conference of the IEEE Engineering in Medicine and Biology, Vol. 7 (Shanghai), 5268-5271. doi: 10.1109/iembs.2005.1615668

Ying, Z., Jia, W., Xinyu, C., and Qiushi, R. (2007). “The processing and control of neuro-stimulator for visual prostheses based on FPGA," in Proceedings of the 2007 IEEE/ICME International Conference on Complex Medical Engineering (Beijing), 1254-1257. doi: 10.1109/ICCME.2007.4381944

Zbrzeski, A., Bornat, Y., Hillen, B., Siu, R., Abbas, J., Jung, R., et al. (2016). Bio-inspired controller on an fpga applied to closed-loop diaphragmatic stimulation. Front. Neurosci. 10:275. doi: 10.3389/fnins.2016.00275

Zhou, Y.-X., Wang, H.-P., Bao, X.-L., Lü, X.-Y., and Wang, Z.-G. (2016). A frequency and pulse-width co-modulation strategy for transcutaneous neuromuscular electrical stimulation based on sEMG time-domain features. J. Neural Eng. 13:016004. doi: 10.1088/1741-2560/13/1/016004

Conflict of Interest: The authors declare that the research was conducted in the absence of any commercial or financial relationships that could be construed as a potential conflict of interest.

Publisher's Note: All claims expressed in this article are solely those of the authors and do not necessarily represent those of their affiliated organizations, or those of the publisher, the editors and the reviewers. Any product that may be evaluated in this article, or claim that may be made by its manufacturer, is not guaranteed or endorsed by the publisher.

Copyright (c) 2022 Mercado-Gutierrez, Dominguez, Hernandez-Popo, QuinzañosFresnedo, Vera-Hernandez, Leija-Salas and Gutierrez-Martinez. This is an openaccess article distributed under the terms of the Creative Commons Attribution License (CC BY). The use, distribution or reproduction in other forums is permitted provided the original author(s) and the copyright owner(s) are credited and that the original publication in this journal is cited, in accordance with accepted academic practice. No use, distribution or reproduction is permitted which does not comply with these terms. 